\title{
Increasing airport capacity utilisation through optimum slot scheduling: review of current developments and identification of future needs
}

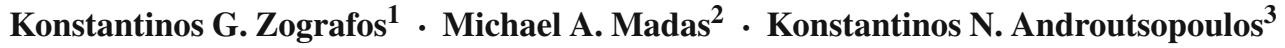

Published online: 22 September 2016

(c) The Author(s) 2016. This article is published with open access at Springerlink.com

\begin{abstract}
Most of the busiest airports worldwide experience serious congestion and delay problems which call for some immediate capacity and demand management action. Solutions aiming to manage congestion through better slot scheduling have lately received a great deal of consideration due to their potential for delivering quick and substantial capacity utilisation improvements. A slot scheduling approach brings promises to cope better with congestion problems in the short to medium run and in a more sustainable way based on existing resources. This paper aims to provide a critical review of current research in declared capacity modelling and strategic slot scheduling. Furthermore, it goes beyond the critical review of current research developments by identifying future research issues and gaps and developing concrete directions towards modelling and solving advanced single airport and network-based slot scheduling problems. Our research findings suggest that the next generation of slot scheduling models should explore variations
\end{abstract}

Paper based on the plenary speech given by Prof. K. G. Zografos at the 10th International Conference on the Practice and Theory of Automated Timetabling (PATAT 2014).

Konstantinos G. Zografos

k.zografos@lancaster.ac.uk

Michael A. Madas

mmadas@uom.gr

Konstantinos N. Androutsopoulos

kandro@aueb.gr

1 Department of Management Science, Lancaster University Management School, Lancaster, UK

2 Department of Applied Informatics, University of Macedonia, Thessaloníki, Greece

3 Department of Management Science and Technology, Athens University of Economics and Business, Athens, Greece of currently used objectives (e.g., alternative expressions of schedule delay) and most importantly enrich them with fairness and equity, resource utilisation and environmental considerations. Future modelling efforts should also aim to further investigate airlines' utility of alternative slot allocation outcomes, including various acceptability measures and levels of tolerance against schedule displacements. Last but not least, future research should intensively focus on the development and validation of computationally viable and robust slot scheduling models being able to capture the complexity, dynamic nature and weather-induced uncertainty of airport operations, along with hybrid solution approaches being able to deal with the size and complexity of slot allocation at network level.

Keywords Airport slot allocation - Airport demand management - Resource-constrained scheduling · Resource allocation $\cdot$ Capacity planning

\section{Introduction}

Air transport plays a significant role for the European economy since it transports $8.8 \%$ of passengers ( 575 billion pkm) and $0.1 \%$ of freight ( 3 billion tkm), representing $22.8 \%$ of EU27 trade by value with the rest of the world (748 billion Euros) in 2011 (European Commission 2013). The rapid growth of demand for air transport services, along with political, physical and institutional constraints on building new airport capacity, has led to acute airport congestion problems with substantial effects on delays. Almost one third of flights in the ECAC area were delayed in 2013, with the average delay per delayed flight exceeding $26 \mathrm{~min}$ (Eurocontrol 2014). Schedule disruptions and delays, especially at busy airports, have a multiplier effect by propagating further into 
the airport network. On top of operational bottlenecks and passenger dissatisfaction, congestion and delays have a major economic and environmental impact spread over the entire air transport system. Air Traffic Management (ATM) inefficiencies in the European Union led to 10.8 million minutes of flight delays in 2012 , costing $€ 4.5$ billion to airspace users and $€ 6.7$ billion to passengers, and producing 7.8 million tonnes of wasted $\mathrm{CO}_{2}$ (IATA 2014a).

The primary cause of these problems is a serious mismatch between growing demand and scarce airport supply. Currently, demand exceeds by far capacity at the busiest European airports for the most part or even throughout the day (Steer Davies Gleave 2011). Unfortunately, there is much evidence that the observed mismatch is further magnified by serious mismanagement and misuse of available airport capacity (Madas and Zografos 2008, 2010; Steer Davies Gleave 2011). A pure supply-side solution through capacity expansion/enhancement is capital intensive and has a long-term horizon of implementation. On the other hand, demand management solutions may lead to quick, incremental improvements in the short and medium run by controlling the spatial or temporal distribution of demand.

Demand management has been long recognised as a principal instrument to deal with capacity shortfalls and delay phenomena in transportation systems (OTA 1984; Fisher 1989; Zografos and Martinez 1990; Saleh 2007; Airports Commission 2013). Demand management has been operationalised in air transport in several variations ranging from pure administrative instruments, such as the spatial reallocation of demand to "reliever" airports or even other transport modes, to market-based measures and slot scheduling approaches. The policy and research community has recently placed the focus on two alternative (and potentially complementary to each other) directions (de Neufville and Odoni 2003): (i) approaches introducing market-driven or pure economic instruments (e.g., slot trading, auctions, congestion pricing) aiming to allocate capacity among competing users by considering real market (or approximations of) valuations of access to congested airport facilities and (ii) efforts aiming to improve the efficiency of the IATAbased allocation mechanism from a slot scheduling point of view. The former category (e.g., market-based/economic instruments) paves the way to a research direction that is strongly based on economic theory, hence going beyond the slot scheduling scope and focus of this paper. The latter category (i.e., slot scheduling) refers to an application of the well studied resource-constrained project scheduling problem (Brucker et al. 1999) in the air transport context. It particularly aims to allocate a certain number of resources (i.e., slots) to dependent activities (i.e., arrivals/departures) over time, while simultaneously satisfying a number of hard and soft constraints (e.g., resource assignment, time assignment, capacities, continuity) (Burke et al. 1997, 2006).
The air transport scheduling problem at hand pertains to the allocation of scarce airport resources (declared airport capacity expressed in slots) for use by airlines into a specific date and time interval. At the strategic level, representing the focus of this paper, there is a proactive schedule coordination process (few months before operations) with bilateral communications between the airlines and the schedule/slot coordinator and several rounds of revisions or improvements of the slot allocation outcome. This process aims to satisfy airlines' requests for particular slots at the maximum possible extent with the ultimate goal of building a feasible flight schedule at both airport and network level, subject to capacity and other operational constraints. Tactical and operational scheduling problems use the schedule developed at the strategic level as input in order to diagnose traffic changes, adjust in real time actual aircraft flows in response to actual operational conditions (e.g., weather) and assign ATM resources (e.g., fleet assignment, gate assignment, aircraft sequencing, allocation of en route slots) on the basis of actual traffic volumes. The strategic IATA-based schedule coordination process (discussed in Sect. 3) represents the dominant mechanism for allocating airport slots at most of the busiest airports worldwide, with some specific adaptations or complements applied at EU airports. The US scheduling practice is different on the grounds that it does not follow the IATA-based scheme for scheduling flights. With the exception of a few airports (New York region airports, Washington/Reagan and Chicago/O'Hare formerly known as HDR airports) that are subject to some scheduling limits, demand is not administered at US airports, while airlines schedule their flights on a wait-based regime, that is, by considering the expected delays.

Slot scheduling signifies a challenging stream of research due to its potential for delivering quick capacity utilisation improvements in conjunction to the complexity and size of the emerging mathematical problems. Slot scheduling models can build upon or complement the existing time-consuming IATA scheduling procedures, being therefore simple, immediately implementable and not requiring organisational or regulatory amendments. From a different perspective, strategic slot scheduling has a strong influence on subsequent resource scheduling options at the tactical and operational level (e.g., fleet assignment, crew scheduling, gate assignment, aircraft sequencing, ground holding/delay programs) due to the inherent interdependencies between strategic planning, tactical adjustments and real time operational interventions in air traffic management (Barnhart et al. 2012). A useful review of the various objectives of different types of stakeholders (e.g., air traffic control, airlines, airport, government) for the singleairport scheduling problem at the tactical/operational level, including constraints and optimisation techniques, is provided by Bennell et al. (2013). The focal research area 
Table 1 Glossary of key terms

Term Description/definition

Airport designation

Coordinated/facilitated airport

Coordination time interval

Declared capacity

Grandfather rights

New entrant

Schedule delay

Series of slots

Slot

Slot coordinator

Slot misuse

Use-it-or-lose-it rule

Weight-based landing fees
The process of designating an airport as "schedule coordinated" or "schedule facilitated" after deeply exploring, in consultation with all airport users, operators and regulators, the opportunities for increasing capacity. The airport designation exhibits certain differences between the relevant EU regulation and the IATA guidelines applied at airports worldwide

A (schedule) coordinated airport is an airport, in which in order to land or take-off, during the periods for which it is coordinated, it is necessary for an air carrier to have a slot allocated by a coordinator. A (schedule) facilitated airport is an airport, where a coordinator has been appointed to facilitate the operations of air carriers operating or intending to operate at that airport

The unit of time (for example, 5, 15 or $60 \mathrm{~min}$ ) used to specify declared capacity levels

At coordinated airports, the competent authorities shall determine the capacity available for slot allocation (i.e., an artificial measure of capacity expressing the number of slots available for allocation per unit of time) twice yearly in cooperation with relevant stakeholders (e.g., ATC, airlines, airport coordinator) according to commonly recognised methods

Grandfather rights stand for historical slot holdings and signify the right to dispose of a slot of the coming flight schedule period, if it has been already used by the respective airline in the current period

A new entrant is defined as an airline requesting a series of slots at an airport on any day, where if the airline's request was accepted, it would hold fewer than five slots at that airport on that day. Half of the slot pool should be necessarily allocated to new entrants

A measure of the difference between the requested and the allocated slot times to airlines

A series of slots is defined as at least five slots having been requested by an airline at an airport for the same time on the same day of the week regularly in the same period and allocated in the same way, or if that is not possible, allocated at approximately the same time. The expression "same time" in this context means within the same coordination parameters (interval)

The permission given to a carrier to use the full range of airport infrastructure necessary to operate an air service at a slot-controlled (schedule coordinated) airport on a specific date and time for the purpose of landing or take-off

A natural or legal person with detailed knowledge of air carrier scheduling coordination is appointed as airport (slot) coordinator of a coordinated or facilitated airport. The same coordinator may be appointed for more than one airport. The slot coordinator is responsible for the allocation of slots and the monitoring of slot usage and shall act in a neutral, non-discriminatory, and transparent way

A number of slots initially allocated but not eventually operated as initially planned (if any at all) at the end of the scheduling season. Typical slot misuse patterns are the following: late return of unwanted slots, flights operated significantly and repeatedly off slot times ("off slot"), failure to operate allocated slots ("no shows"), and flights operated without having cleared slots ("go shows")

It represents a minimum slot usage rule based on which, “...if the $80 \%$ usage of the series of slots cannot be demonstrated, all slots constituting that series shall be placed in the slot pool, unless the non-utilisation can be justified on the basis of [a number of. . .] reasons"

A measure of the aircraft weight (i.e., maximum take-off weight) that is used to calculate (proportionally) the landing fees for a given flight of this paper is the strategic level of airport capacity allocation through the setting of optimum declared capacity levels and slot scheduling. The objective of this paper is threefold: (i) to provide a critical review of the role of declared capacity modelling and strategic slot scheduling in optimising the allocation and use of scarce airport resources, (ii) to identify emerging research issues and gaps between currently available methods or models and emerging decision support needs and (iii) to propose concrete directions for future research in strategic airport slot scheduling.
The remainder of this paper is structured into five sections. Section 2 presents the review approach, and Sect. 3 provides a brief description of the current slot allocation practice along with the underlying problems and inefficiencies. Section 4 presents an overview of the major thrust and evolution of relevant research on two prominent streams related to declared capacity and slot scheduling. Section 5 discusses the emerging modelling needs and suggests future research directions, and Sect. 6 presents the key concluding remarks of the paper. The paper is complemented by a glossary of key terms (Table 1) and a list of acronyms (Table 2). 
Table 2 List of acronyms

\begin{tabular}{ll}
\hline ACI & Airports Council International \\
ATFM & Air Traffic Flow Management \\
ATM & Air Traffic Management \\
CPU & Central Processing Unit \\
ECAC & European Civil Aviation Conference \\
EU & European Union \\
GFR & Grandfather Rights \\
HDR & High Density Rule \\
IATA & International Air Transport Association \\
IFR & Instrument Flight Rules \\
pkm & Passenger-kilometres \\
tkm & Tonne-kilometres \\
\hline
\end{tabular}

\section{Review approach}

The review approach is divided into two parallel streams of research addressing: (i) the analysis of the current state of practice (discussed in Sect. 3) and (ii) the review of relevant research on capacity and slot allocation modelling (presented in Sect. 4). The analysis of current practice examines the relevant regulatory background, policy priorities and debates, slot allocation procedures, as well as scheduling rules and guidelines. It involves the review of: (i) relevant policy documents and studies, such as the IATA Worldwide Slot Guidelines (IATA 2014b), as well as the complementary EU Regulation 95/93 and its several amendments (European Commission 1993, 2004, 2009, 2011) and (ii) several studies and technical reports (e.g., PwC 2000; TUB 2001; NERA 2004; Mott MacDonald 2006; Steer Davies Gleave 2011) mainly dealing with the assessment of the state of implementation of Regulation 95/93 across EU Member States, the identification of problems and areas of improvements, as well as the investigation of the potential acceptability and impacts of market-based mechanisms. The review of relevant academic literature focuses on declared capacity and slot allocation modelling research. Relevant literature was searched through keywords such as airport slot allocation, slot scheduling, declared capacity, airport schedule optimisation, demand and capacity management, as well as airport congestion and delays. Overall, this resulted in a comprehensive review of 96 research papers (almost two thirds cited in the paper) published in a long list of widely recognised scientific journals in the following knowledge areas: (i) (air) transportation, (ii) economics and (iii) operations research and management science.

Figure 1 presents the numbers of relevant research papers as well as policy documents and studies published between 1993 and 2014. It can be observed that the publication of the EU Regulation 95/93 (or "The Regulation") "on common rules for the allocation of slots at Community airports" on 18 January 1993 (European Commission 1993) has triggered a wealth of research that peaked during the last decade due to the long-awaited drastic revision of current slot allocation practices. Interestingly, although numerous papers lie at the intersection of scheduling models and market-based allocation mechanisms, an increasing body of research seems to be concentrated on strategic slot scheduling during the last six to seven years.

The identified research papers were reviewed with special focus on slot allocation modelling objectives, formulation and solution approach (including trade-offs between modelling accuracy and efficiency of the solution). The integrated analysis of the identified decision-making requirements, problems and inefficiencies in current allocation practice in conjunction to existing methods and models drawn from research literature eventually leads to the identification of emerging research issues and gaps that are discussed in Sect. 5.

Literature Review Statistics

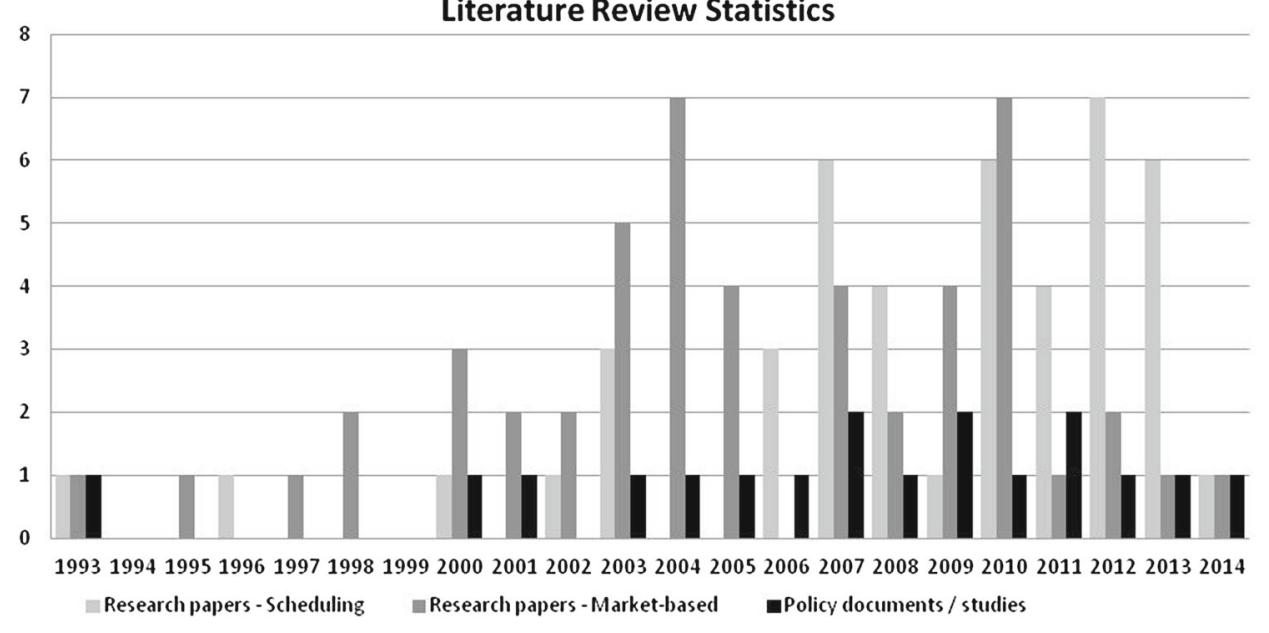




\section{Current practice}

Capacity at about 170 of the busiest and most congested airports worldwide (around 100 in Europe) is expressed and allocated in slots. A slot identifies a time interval, specific date and time, during which a carrier is permitted to use the airport infrastructure for landing or take-off at a slot-controlled (coordinated) airport (European Commission 1993). Slots are allocated according to a complicated set of administrative rules, criteria and priorities within a framework of voluntary guidelines developed and evolved over the years under the auspices of IATA (IATA 2014b) with local interpretations and adaptations. Since 1993, the IATA-based slot allocation system has been adapted, complemented and further updated or amended by European Union regulation (European Commission 1993, 2004, 2009, 2011) for EU airports.

According to the IATA-based system and the complementary EU regulation, a long list of slot scheduling guidelines set out administrative procedures, define allocation rules, criteria and priorities (e.g., historical usage or "grandfather" rights, "use-it-or-lose-it" rule, "new entrant" rules), designate coordinated (slot-controlled) airports, and appoint schedule coordinators in order to accommodate and monitor the airlines' slot requests and utilisation at local airport level. These guidelines and rules coordinate the slot scheduling process at each airport, with the airlines implicitly undertaking the complicated task of effectively matching slots at their airport network. In order to cope with strong slot complementarity at network level, the IATA-based system provides for the organisation of biannual, worldwide scheduling conferences aiming to match slots and eventually improve-through bilateral negotiations-the final slot scheduling outcome.

A critical issue in slot allocation is the determination of declared airport capacity which practically controls the number of slots available for allocation per unit of time. Slot scheduling and the setting of optimum declared capacity levels are closely interdependent and both lie at the heart of optimising the allocation and use of scarce airport resources. Slot scheduling procedures currently in use are simplistic in representing the various objectives and operational or regulatory constraints of the affected stakeholders and they employ empirical or ad hoc processes for determining (rather than computing) declared capacity. Moreover, difficulties and limitations in current allocation practice for single airports feed into the hugely complex network-wide problem. Overall, the existing slot scheduling process does not address the complexities of the real-world problem in a realistic manner and applies an oversimplified approach that is eventually at the expense of allocation efficiency and utilisation of scarce airport resources. Poor allocation outcomes imply an inherent failure to properly match requested slots with those eventually allocated to airlines. In addition, slot misuse (e.g., late return of unwanted slots, "off slot", "no shows") sharpens the capacity shortage due to poor utilisation of a resource already running into scarcity (ACI Europe 2004). Even at airports where slot demand exceeds capacity, over $10 \%$ of the allocated slots go unused (Steer Davies Gleave 2011). ACI Europe 2009 estimated that slots unused due to their late return account for losses of around $€ 20$ million per season at large, congested European airports.

Allocation inefficiencies and slot misuse problems are further amplified when one considers the strong interdependencies in the allocation of various resources (e.g., airport slots, airspace sectors) at the strategic, tactical and operational level. In practice, airport slots are allocated at the strategic planning phase that is initiated some few months before operations. However, during the tactical and operational phase (a couple of days to a few hours before operations), airport slots are not effectively taken into account. Airport slots, allocated at the strategic level, are only viewed as a planning parameter for airports but are not sufficiently linked to flow management and the allocation of airspace capacity at tactical and operational levels (Eurocontrol 2013). This reduces the predictability of traffic at operational level and often creates under- or over-deliveries in airspace capacity (i.e., declared capacity is exceeded by the actual number of aircraft entering an ATM sector during a particular period). In any case, dealing with strategic slot scheduling problems needs to be treated not only as an end in itself but also as a major influential process to strongly interdependent scheduling problems at other airports (nodes), airspace sectors (links) or even ATM planning stages.

A number of decision-making requirements emerge from the various problems, limitations and inefficiencies in existing capacity allocation practice:

- There is a need to apply a more sustainable and effective approach to cope with capacity shortages in the short run with the use of existing resources. Solutions aiming to manage congestion through the optimum scheduling of airport slots are clearly compatible with this need.

- Available capacity should be better exploited in terms of utilisation rates. This calls for remedies and measures targeting both the demand-capacity imbalance and slot misuse problems. Close monitoring of actual slot utilisation along with a systematic control of consistency in slot use across all planning stages (e.g., strategic, tactical, operational) needs to be ensured or even reinforced (e.g., sanctions for intended and repeated misuse).

- Allocation inefficiencies are closely linked to misuse problems. It is reasonable to expect that slots that are better matched to airlines' requests are more acceptable and, most importantly, will be more intensively used during the operational phase. It is therefore necessary to obtain better insight into airlines' utility (or dis-utility) of alter- 
native slot allocation outcomes, including measures and levels of tolerance against deviations from requested slot times as well as their real economic valuation of slots.

- There is a need to rationalise the definition and setting of declared airport capacity at appropriate levels. In the process of setting optimal declared capacity levels, it should be taken into account that capacity determinant factors may change substantially over time (e.g., adverse weather conditions, unforeseen events), thus making necessary a dynamic approach for the management of capacity.

- A realistic representation of the objectives, constraints and preferences of the various stakeholders involved in or affected by the slot allocation process is needed.

- Viable and feasible flight schedules should be ensured for airlines, individual airports and the overall ATM system throughout the entire ATM planning lifecycle.

- Advanced decision support capabilities and tools need to be developed to cope with the size and complexity of slot allocation, both at single-airport and especially at airport network level.

At the outset, the existing slot allocation process is quite far from perfect. There is large room for improving allocation efficiency by adopting a more realistic representation and modelling of capacity determination and the interrelated slot allocation problems. Based on different assumptions and orientations, recent research efforts suggest some interesting advances in the underpinning mathematics of slot scheduling. In specific, they develop and investigate optimisation models and efficient solution algorithms for the large-scale, complex airport capacity allocation problem. These are extensively discussed in what follows.

\section{Overview of slot allocation research}

The IATA-based slot allocation scheme (Fig. 2) envisages three distinct methodological steps, each involving different research challenges and feedback loops (indicated with dashed lines): (i) pre-allocation, (ii) allocation and (iii) postallocation activity. The pre-allocation activity is initiated well in advance of operations and involves research topics dealing mainly with the proper definition, setting and rationing of declared airport capacity. Although declared capacity modelling constitutes an inherent problem in slot allocation modelling, we dedicate a separate subsection (Sect. 4.1) to discuss relevant research considerations due to the strongly influential role of declared capacity as input parameter or constraint to slot scheduling. Having set declared capacity levels, the core allocation phase (few months before operations) matches slot requests with slots eventually allocated to airlines. This matching is based on a certain slot valuation that leads to a quantity (e.g., slots allocated as compared to those requested) and quality (i.e., "distance" from original slot requests) of allocated slots.

Two alternative research approaches are proposed in literature to allocate capacity among competing users and assign some valuations on scarce airport capacity: (i) market-based or pricing instruments and (ii) slot scheduling approaches. Market-driven or pure pricing mechanisms aim to assign some explicit value on slots and allocate capacity by considering real market (or approximations of) valuations of access to congested airports. In a pure pricing form, congestionbased pricing schemes have been examined as an access control mechanism in order to handle the temporal profile of demand through congestion-based fees (Brueckner 2002; Morrison and Winston 2007). Market-based mechanisms for capacity allocation have also received a great deal of consideration in the policy and research community. Auctioning part of or the entire slot pool complemented by trading at the secondary market has been extensively addressed in literature (Mott MacDonald 2006; NERA 2004; TUB 2001). Other researchers (Madas and Zografos 2006, 2008; Ranieri et al. 2013) have examined individual mechanisms or instruments in order to define and assess the potential performance of comprehensive slot allocation frameworks integrating market-driven or economic options at varying degrees of implementation (e.g., primary/secondary trading, auctioning part or the entire slot pool). Market-driven or pricing instruments discussed above are both promising and potentially efficient in dealing with serious congestion problems at airports where demand exceeds capacity throughout the day. However, their modelling techniques and underlying principles stem mainly from economic theory hence going beyond the slot scheduling scope of this paper. On the other hand, slot scheduling approaches involve the modelling of the slot allocation process as a scheduling problem (i.e., single-airport, airport network) with different underlying criteria and objectives (e.g., operational efficiency, allocation efficiency, resource utilisation, environmental impacts, equity, fairness). Key research developments and challenges in relevant strategic slot scheduling research are presented in Sect. 4.2.

The post-allocation phase involves the monitoring of slot utilisation and compliance while simultaneously providing feedback (i.e., dashed lines) for potential demand readjustments or re-assessment of declared capacity on the basis of actual operational patterns and experiences (e.g., weather conditions, actual traffic volumes, utilisation patterns). As part of the post-allocation phase, a number of interrelated tactical and operational scheduling problems emerge. On the basis of the schedule developed at the strategic level, tactical and operational scheduling problems aim to model how actual aircraft flows are adjusted in response to actual operational conditions (e.g., weather) and assign various ATM 
resources on the basis of actual traffic volumes. Typical problems under this category are runway scheduling (Bennell et al. 2013), fleet assignment (Papadakos 2009; Pita et al. 2014), gate assignment (Dorndorf et al. 2007, 2016; Haghani and Chen 1998; Narciso and Piera 2015), aircraft sequencing (Bianco et al. (1997); Caprl and Ignaccolo (2004)), crew scheduling (Ball et al. 2007; Papadakos 2009), as well as the ATFM ground holding/delay programs (Andreatta et al. 1995; Ball et al. 2007). Tactical and operational scheduling problems and the inherent dependencies between strategic planning, tactical adjustments and real time operational interventions in air traffic management have been extensively discussed in relevant literature (Ball et al. 2007; Barnhart et al. 2012) and will not be addressed further in this paper.

\subsection{Declared capacity modelling}

Declared capacity constitutes a fundamental concept in the existing IATA-based slot scheduling scheme. It specifies the number of slots available for allocation at a slot-controlled airport per unit of time and is most frequently expressed in terms of scheduled movements (landings and take-offs) during each coordination time interval. The coordination time interval represents the unit of time used as the basis for capacity determination and slot allocation. Declared capacity is further specified by a set of rolling constraints which restrict the number of arrivals, departures and total movements that can be accommodated within different time intervals in order to control the concentration of traffic throughout the day. The determination of capacity is normally tied with the capacity of the runway system. However, other criteria may also apply, including the terminal capacity for passengers of different movement types (departure/arrival) and flight types (Schengen/non-Schengen, domestic/international), the availability of apron stands for each aircraft type and other factors such as noise restrictions and night curfews (Eurocontrol 2005, 2009).

Declared capacity represents an administrative measure of capacity dependent on maximum throughput. In theory, it is determined by the slot coordinator at each slot-controlled airport with certain assumptions and attributes and following a comprehensive capacity assessment study taking into account various capacity determinants (Ball et al. 2007) (refer back to Fig. 2). In practice, however, declared capacity is typically set to $85-90 \%$ of maximum throughput (de Neufville and Odoni 2003). Once defined, it is then rationed according to a set of criteria for assigning different priorities to requests for slots. Under the current slot regime (IATA 2014b), these criteria can be thought of as classifying requests into the following three general priority classes: (i) requests with historical rights ("grandfathered slots"), (ii) requests with new entrant status and (iii) all remaining requests. Within each class, additional criteria may also apply, resulting in multiple subclasses with different priorities.

Despite its critical importance and its substantial influence on the efficiency of the allocation process, the declared capacity determination process has not been sufficiently examined (Railsback and Sherry 2006). Koesters (2007a) examined the real use of allocated and agreed slots at the actual day of operations and demonstrated the poor utilisation rates and a serious misuse of allocated slots at coordinated German airports. Other researchers (Zografos et al. 2012) have shown that increases in the order of $20 \%$ in declared capacity lead to substantial improvements in the allocation efficiency (i.e., better matching of requested with allocated slots) in the order of $65 \%$. The current practice on the determination of declared capacity in slot coordinated airports has received much criticism (Ball et al. 2007; Odoni and Morisset 2010) with main concerns being the following: (i) there is ample room for local interpretations and adaptations, (ii) setting of very low capacity levels as a percentage of the capacity under Instrument Flight Rules (IFR), (iii) the process for determining the declared capacity is empirical or ad hoc, and (iv) there is limited consultation with stakeholders. At the outset, this criticism focuses on the following two major categories of issues: (i) the lack of a harmonised and commonly agreed way of interpreting, determining and managing declared capacity and (ii) the setting of declared capacity at levels that are too high or too low. Barnhart et al. (2012) identified, as one of the strategic research priorities for the next decade, the need to use analytical modelling in order to appropriately specify and allocate airport capacity, while simultaneously analysing the trade-offs and implications of over-scheduling and underscheduling. The lack of a harmonised view of the complex declared capacity construct can be confronted with a careful and commonly agreed definition of the following attributes and underlying assumptions:

- Fixed versus variable levels: declared capacity typically refers to a constant number of slots per unit of time but time-varying levels can be also set to take into account the changing mix and distribution of arrivals and departures in a daily flight schedule.

- Level of aggregation: the typical metric of declared capacity pertains to the number of available slots per time interval. However, due to the fluctuation of declared capacity throughout the day, alternative declared capacity indicators have also been developed (e.g., daily, entire scheduling season) (Eurocontrol 2009).

- Type of movement: the declared capacity may be broken down and set limits for arrivals, departures and total movements. The applied ratio of departures over arrivals plays a decisive role on the specification of declared capacity limits for different types of movements. 


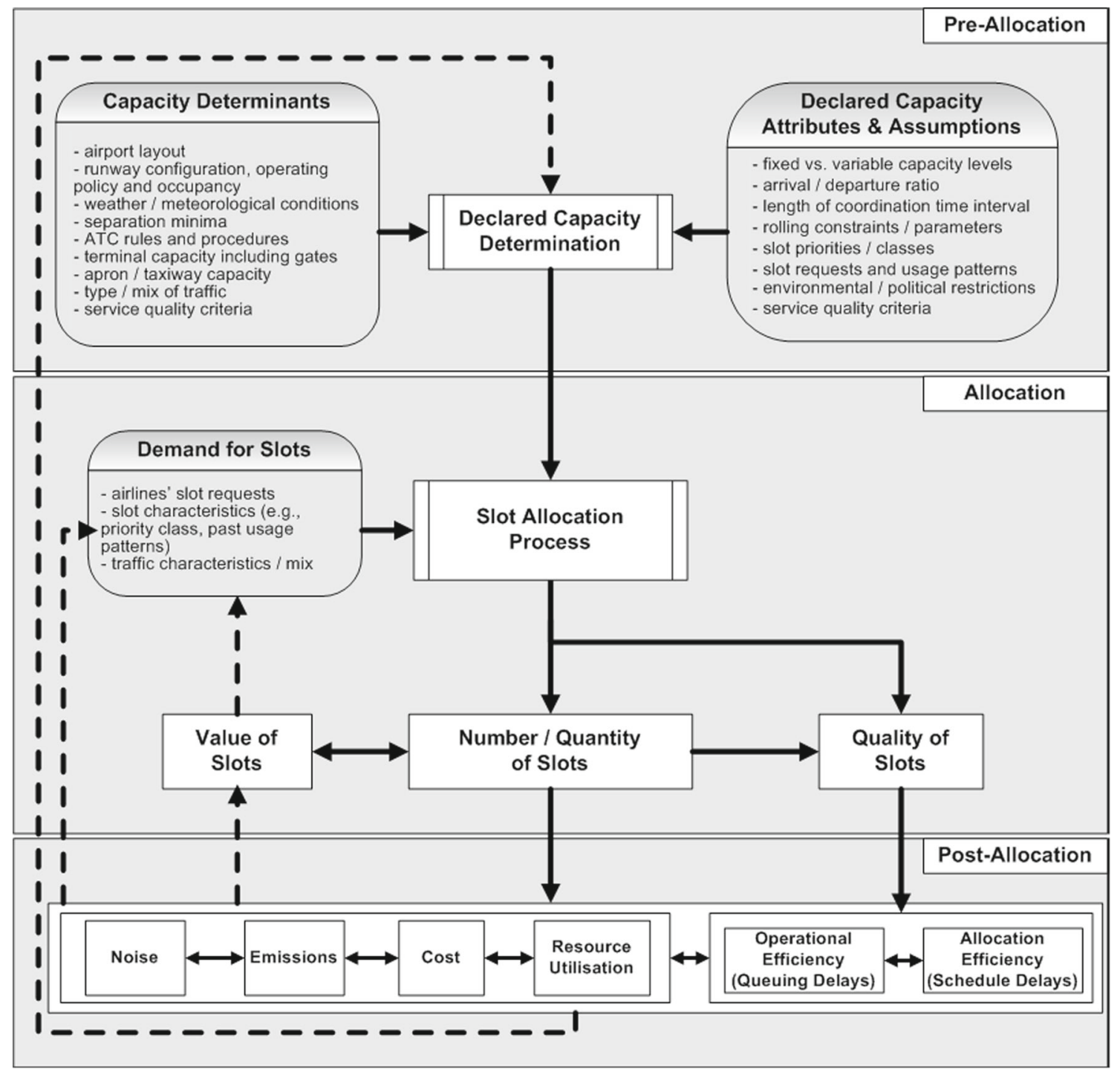

Fig. 2 Problem scope

- Coordination time intervals: the unit of time (e.g., 5, $15,60 \mathrm{~min}$ ) used for slot coordination purposes does not only affect the size and complexity of the slot scheduling problem but also the actual operations (e.g., a long time interval may encourage some "bunching" of flights).

- Rolling capacity constraints: various rolling parameters are used to control the concentration of demand in different time intervals and ensure that capacity saturation conditions do not persist for a long time within the day. The selection of the rolling capacity constraints affects the formulation, and most importantly the complexity and accuracy of the scheduling problem.

- Capacity determinant factors: typical critical factors can be outlined as follows: (i) runway capacity under variable weather/meteorological conditions, (ii) terminal capacity, including gates, (iii) apron/taxiway capacity, (iv) type/mix of traffic (e.g., wake vortex aircraft categories), (v) environmental considerations ("environmental capacity") and/or political restrictions (e.g., noise limits, night curfews, cap on annual movements), and (vi) service quality parameters (e.g., average delay) (Eurocontrol 2009).

- Reference capacity metric: the declared capacity is dependent on reference capacity metrics which are usually driven by runway capacity shortages. However, there is no consensus about the runway capacity that should be used as a reference capacity figure for deriving the declared capacity measure (usually in the form of a percentage of the reference capacity). Maximum throughput, saturation capacity, nominal, optimal, maximum sustainable, unconstrained, sustained capacity, operational capacity and service rates are some indicative examples 


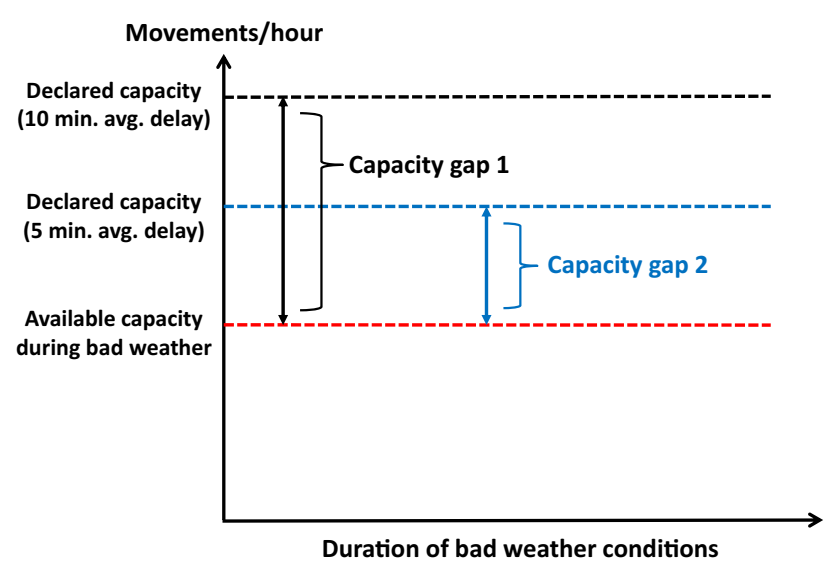

Fig. 3 Weather dependency in declaring airport capacity (Eurocontrol 2005)

of metrics (some of which with overlapping interpretations) that are used to infer or derive declared capacity levels (Eurocontrol 2005, 2009).

- Weather dependency: airports are vulnerable to bad weather, and capacity varies strongly with changing weather conditions. Declared capacities at EU airports are largely determined by their IFR capacities. In order to deal with weather-related disruptions, some airports apply more sustainable capacity levels such as a modest (low) estimate of declared capacity accounting for long delays (Odoni and Morisset 2010). In any case, weatherinduced uncertainty and variability of airport capacity needs to be considered in setting declared capacity levels with special view to effects on schedule reliability and punctuality (Ball et al. 2007; Odoni and Morisset 2010). Furthermore, the actual capacity reduction during bad weather operations is also influenced by the assumed service quality criterion (e.g., acceptable delay) in declaring airport capacity. Airports adopting a rather optimistic estimate of capacity by assuming high average delays will be more severely affected (capacity gap 1) during adverse weather as compared to airports which have factored-in a lower portion of delay (capacity gap 2) (Fig. 3) (Eurocontrol 2005).

From a long-term research perspective, the efficient specification of system-wide capacities with stochastic capacity considerations due to weather dependencies and the analysis and mathematical modelling of the implications of overscheduling and under-scheduling constitute challenges for future research. Barnhart et al. (2012) stressed the need for a comprehensive evaluation of the impact of different levels of capacity specification that will, among other things, provide insights into the optimal trade-off between schedule and queuing delay. As a matter of fact, the level of declared capacity has serious impacts on the allocation efficiency, and at the

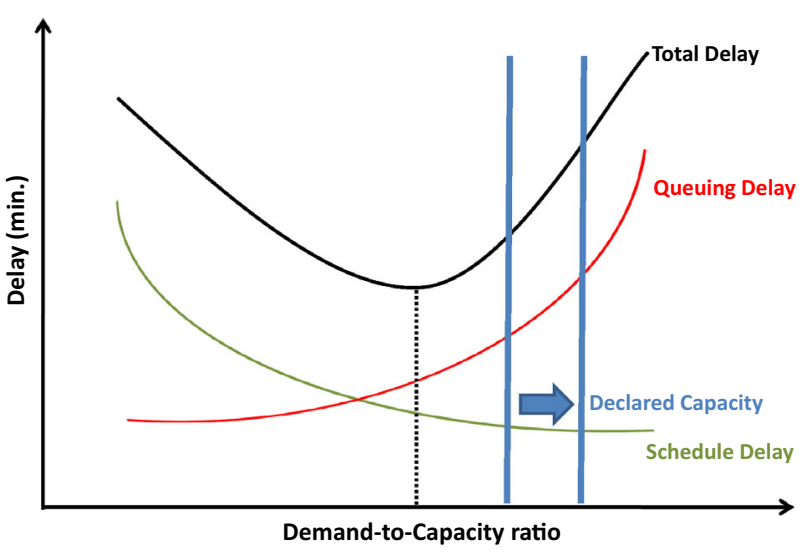

Fig. 4 Declared capacity, schedule and queuing delay (adapted from Swaroop et al. (2012))

same time, it affects the classical trade-off between acceptable level of service and utilisation levels of scarce airport resources (Swaroop et al. 2012) (Fig. 4). As far as the allocation efficiency is concerned, the level of declared capacity represents essentially the degree to which the allocation problem is constrained and, consequently, the potential ability of the allocation mechanism to satisfy airlines' requests for slots by properly matching slots requested with slots eventually allocated to airlines ("schedule delay"). In this respect, it has been demonstrated that an increase in declared capacity (shifting blue vertical line to the right) improves disproportionately the allocation outcome, with the opposite being also true (Zografos et al. 2012). As a result, the level of declared capacity basically determines not only the number of slots available for allocation to airlines, but also the quality of slots in terms of the "distance" from their requests. On the other hand, there is a clear trade-off between declared capacity levels and operational efficiency ("queuing delay"). Higher declared capacity leads to increased frequency, competition, access and ultimately utilisation of scarce infrastructure, often at the expense of queuing delays ("delay" measure) and schedule reliability.

An interesting research direction that has been recently investigated and can provide substantial input to both declared capacity and slot allocation modelling relates to the determination of optimal scheduling limits ("slot caps" in U.S. or declared capacity levels in Europe) with dynamic and stochastic capacity considerations, as well as the explicit modelling of the slot value for performing an operation at congested airports. Churchill et al. (2012) developed a stochastic optimisation model to determine the optimal, timevarying number of slots that should be made available for allocation at a certain (single) airport, taking into account: (i) the long-term capacity uncertainty (i.e., various capacity profile scenarios) due to weather conditions and (ii) different valuations of slots (in economic terms) at different 
Fig. 5 Modelling the effects of declared capacity levels on queuing delays
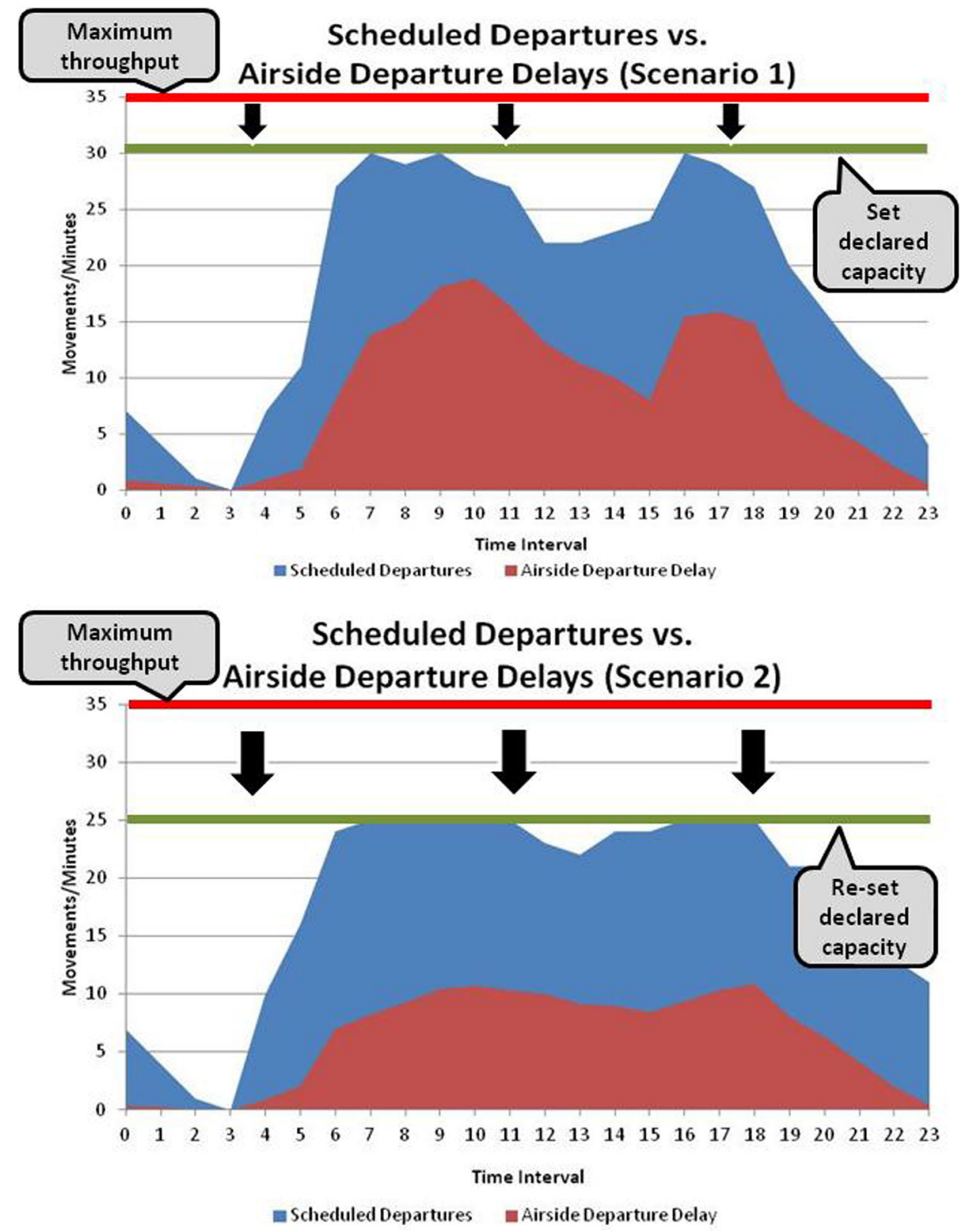

times of the day. Three interesting observations can be made here. First, slot issuance is a long-term, strategic-level decision that is seriously affected on a daily basis by abnormal weather conditions inducing airport operations. Second, the real value of performing an operation at a congested airport varies substantially with time since airlines prefer to operate at certain times of the day for various reasons (e.g., business travellers, flight lengths, time zone changes). Third, despite the attractive simplicity of declaring a fixed number of slots for each time period, time-dependent declared capacity levels are closer to reality, act better as queuing recovery mechanisms and fit with the time-varying profiles of slot values and weather-adjusted capacity levels.
Declared capacity serves also as a "control valve" of operational delays at airports otherwise operated at or close to their maximum throughput for long periods of time (Fig. 5). Being set with reference to maximum throughput (or saturation capacity) (de Neufville and Odoni 2003), it implicitly controls the actual traffic and consequently the associated delays at congested airports through the available number of allocated slots. Reducing, for example, declared capacity levels at a major Greek airport from $85 \%$ (upper part of Fig. 5) to $71 \%$ (lower part of Fig. 5) (or from 30 to 25 hourly departures) of maximum throughput (35 departures) has a notable impact on peak airside departure delays. 
The investigation of the research issues discussed above necessitates the deployment of effective means of modelling capacity along with the assessment of impacts of varying demand and capacity levels on various performance metrics (e.g., delays, noise, emissions). Existing modelling capabilities and tools for airport capacity and delay assessment have been extensively reviewed in the literature (Lucic et al. 2007; Long et al. 2009). Analytical models gradually evolved and were encapsulated into software tools (Malone 1995; Stamatopoulos et al. 2004) or integrated Decision Support Systems (Zografos and Madas 2006; Zografos et al. 2013). Interestingly, some of these models (Pyrgiotis 2011; Pyrgiotis et al. 2013) were extended to capture also "ripple effects" propagating from a local airport to a system-wide basis (i.e., airport network). These can be expanded to support the setting of optimal declared capacity levels both for a single airport and the ATM network as a whole (including airspace sectors).

\subsection{Slot scheduling}

This section defines the slot scheduling problem, highlights its key features and provides a critical review of relevant approaches and models addressing the problem at both single-airport and network level.

\subsubsection{Key features of slot scheduling}

The slot scheduling problem aims to optimally assign (few months before operations) requested movements (requests for landing or take-off) at an airport to coordination time intervals within a given scheduling period. Typical constraints for the slot scheduling problem emerge from the declared airport capacity, the priorities arising from the existing slot allocation framework (e.g., historical slot holdings), the operational requirements of airlines and airports (e.g., turnaround time, ground handling, flight connectivity), ATFM constraints (e.g., airspace sectors' capacity), as well as the criteria used for scheduling slot requests. In existing literature, the slot scheduling problem has been studied in the context of either a single airport (Koesters 2007b; Zografos et al. 2012) or a network of airports (Castelli et al. 2011, 2012; Corolli et al. 2014). Airport capacity is modelled either as a deterministic (Zografos et al. 2012) or a stochastic parameter (Corolli et al. 2014).

A primary criterion of the slot scheduling problem (Koesters 2007b; Zografos et al. 2012; Castelli et al. 2011, 2012; Corolli et al. 2014) is the minimisation of a delaybased cost function. This can be expressed either in the form of typical operational delay or the "schedule delay" concept (Swaroop et al. 2012). Operational delay is usually expressed in terms of the expected arrival/departure delays and total passenger delays. "Schedule delay" is a distance- based measure expressing the difference between requested and allocated slot times and is used as a proxy of the value assigned to a given time interval (slot time) by the airlines. Koesters (2007b) developed a deterministic approach to model the initial slot allocation process and calculated schedule delays for various declared capacity and demand levels.

On top of allocation efficiency considerations (usually expressed by delay measures), fairness and equity, access and competition, as well as environmental objectives have been also proposed by researchers (Cohen and Odoni 1985; Andreatta and Lulli 2009; Ranieri et al. 2013). Such objectives aim to allocate capacity among several classes of users of congested airports by maximising some type of a social welfare function taking into account policy or social considerations (e.g., user's ability-to-pay principle, non-discriminatory allocation practices, access to small communities). In order to maximise social benefits, "socially desirable" criteria are proposed: (i) number of passengers served by the resulting schedule in order to ensure sufficient utilisation of resources, (ii) number of airports or cities served to safeguard connectivity to small communities, (iii) number of slots (including grandfathered) reserved for established airlines to avoid drastic changes at the expense of schedule feasibility and viability, (iv) number of slots allocated to new entrants to ensure equity and a level playing field for all competing airlines and (v) total or average noise exposure or emissions at an airport.

Depending on the geographical scope of the slot scheduling problem, two basic model categories are encountered: (i) single-airport models and (ii) airport network models in which slots are simultaneously allocated at a network of airports.

\subsubsection{Single-airport models}

A slot request issued by an airline usually involves a specific coordination interval for a series of days ranging throughout the scheduling period (e.g., 08:00 AM, every Monday). The single-airport slot scheduling problem aims to allocate series of airport slots (in specific priority classes) to coordination time intervals within a given scheduling period under airport capacity and airports'/airlines' operational constraints. It belongs to the category of resource-constrained scheduling problems where the arrival/departure slot requests constitute the jobs to be processed by a single constrained resource type, i.e., the airport, over the entire planning horizon. The airport capacity represents the available resource units. For a unified notation and classification scheme of resource-constrained project scheduling problems, the reader can also refer to Brucker et al. (1999).

Based on the formulation of the general resource-constrained scheduling problem of Pritsker et al. 
(1969), Zografos et al. (2012) formulated the single-airport slot scheduling problem as an integer linear, deterministic scheduling model. The problem is defined for a set of coordination time intervals $T=\{0, \ldots, n-1\}$ defined per day $\delta \in D$ (the set of calendar days for the scheduling period) and the set of requested (series of) movements $M$. Each movement $m \in M$ is associated with a series of slots over the entire planning horizon, modelled through parameters $a_{m}^{\delta}$, which take value one if movement $m$ operates on day $\delta \in D$, and zero otherwise. A capacity constraint $c \in C$ is defined by the airport capacity values $u_{c}^{\delta t}$ defined for a time period of fixed duration (e.g., $15 \mathrm{~min}$ ) starting at time interval $t$ and day $\delta$. Thus, for a specified start time $s, T_{c}^{s}=\left\{t \in T \mid s \leq t<s+t_{c}\right\}$ defines the set of consecutive coordination time intervals over which the constraint is active. Stated otherwise, the constraint ensures that the total number of movements allocated to time intervals in $T_{c}^{s}$ should not exceed $u_{c}^{\delta s}$ for any $s$ such that $0 \leq s \leq n-t_{c}$. Moreover, capacity constraints may be modelled separately for arrivals and departures through parameters $\left(b_{m c}\right)$, being equal to one if the movement is an arrival/departure and the constraint applies to arrivals/departures only, and zero otherwise. In addition to the capacity constraints, the model proposed by Zografos et al. (2012) incorporates the precedence constraint imposed through minimum turnaround time $t_{a d} \geq 0$ for each pair of movements $\left(m_{a}, m_{d}\right), m_{a}, m_{d} \in M$, such that $m_{a}$ is the arrival that corresponds to departure $m_{d}$. The cost of allocating movement $m \in M$ to coordination time interval $t \in T$ is $f_{m}^{t} \geq 0$. The latter is modelled as $\left|t-t_{m}\right|$, with $t_{m}$ expressing the time interval originally requested for $m$. An indicative formulation (Zografos et al. 2012) of the singleairport slot allocation problem is given by (1)-(4), where the allocation of movements $(m)$ to time intervals $(t)$ is specified through binary decision variables $\left(x_{m}^{t}\right)$, which take value one if movement $m$ is allocated to interval $t$, and zero otherwise.

$$
\begin{aligned}
& \operatorname{minimise} \sum_{m \in M} \sum_{t \in T} f_{m}^{t} x_{m}^{t} \\
& \text { subject to } \sum_{t \in T} x_{m}^{t}=1, \quad m \in M \\
& \sum_{m \in M} \sum_{t \in T_{c}^{s}} a_{m}^{\delta} b_{m c} x_{m}^{t} \leq u_{c}^{\delta s}, \quad c \in C, \quad \delta \in D, \quad s \in T_{c} \\
& \sum_{t \in[0, k)} x_{m_{d}}^{t}+\sum_{t \in\left[k-t_{a d}, n\right)} x_{m_{a}}^{t} \leq 1,\left\{m_{a}, m_{d}\right\} \in P, k \in\left[t_{a d}, n\right) \\
& x_{m}^{t} \in\{0,1\}, \quad m \in M, \quad t \in T
\end{aligned}
$$

The objective function (1) minimises the total absolute difference between the requested and allocated time interval (i.e., "schedule delay"). Expression (2) ensures that every movement is allocated to a single time interval, while (3) specifies that the allocation of movement to a certain time interval must satisfy the corresponding capacity constraints.
The turnaround time constraints (4) resemble those proposed by Christofides et al. (1987) in the context of project scheduling and apply to requests for which both an arrival and a departure slot time have been requested. These constraints enforce the obvious restriction that an arrival must be separated from a departure by at least a specified number of coordination time intervals, expressing the aircraft turnaround time. The number of time intervals usually depends on the aircraft type, accounting for the fact that larger aircraft require larger turnaround (service) times. Although constraint (4) involves a large number of inequalities, the emerging formulation leads to strong linear relaxations.

The single-airport slot allocation problem falls into the category of scheduling problems with time-dependent resource constraints, a generalised version of the resourceconstrained project scheduling problem (Brucker et al. 1999) known to be NP-hard (Garey and Johnson 1979). Consequently, it is rather unlikely to find a solution algorithm with polynomial complexity. Zografos et al. (2012) proposed a heuristic algorithm for the scheduling problem at hand, in which slots are allocated hierarchically for each priority class. This approach is aligned with IATA's fundamental requirement giving the ultimate priority to the satisfaction of slot requests of airlines having "grandfathered rights". After solving the proposed model for slot requests pertaining to a given priority class, capacity constraints are updated accordingly, and the model is solved for slot requests of the subsequent priority class. The linear relaxation of a restricted version of the problem is solved with capacity constraints added iteratively only as needed. If the optimal solution is fractional, then reduce-and-split cuts (Andersen et al. 2005) are generated and included in the model. If the solution remains fractional, then variable fixing is performed by identifying the variable with the highest fractional value and fixing its value to one. Subsequently, the linear relaxation is solved again, and the process iterates until an integer solution is found. The proposed slot allocation model was applied at three international airports in Greece. The largest problem instance involved 1087 slot requests (on a daily basis) for a planning horizon of 197 scheduling days, characterised as a medium-sized problem. For problem instances in which the corresponding linear relaxation of the proposed formulation was strong, the solution time ranged from a few seconds up to $3 \mathrm{~min}$. For instances in which additional cutting planes constraints were used to solve the integer program, the solution time ranged up to four and a half hours, which is, however, clearly compatible with the strategic planning context (i.e., few months before operation) of the respective slot allocation problem.

Jacquillat and Odoni (2015) proposed an integrated approach that jointly optimises the flight scheduling at the strategic level and the utilisation of airport capacity at the tactical level subject to capacity and delay reduction constraints. 
The proposed single-airport scheduling model reflects mostly the US scheduling practice, and it is based on the strategic network-based scheduling model of Pyrgiotis and Odoni (2015)) discussed in the subsequent section. It considers a two-stage lexicographic objective that aims to determine a schedule minimising maximum displacement while simultaneously keeping total (aggregate) displacement at the lowest possible levels. The authors demonstrated the model at John F. Kennedy International (JFK) airport and showed that a moderate rescheduling (no more than 15-30 min per flight) of $75-90 \%$ of the flights can drastically reduce congestion at busy U.S. airports.

\subsubsection{Airport network models}

A strong criticism of the existing slot allocation practice is that it does not explicitly consider the strong complementarity of slots assigned to a single flight at origin and destination airports. This inefficiency of the existing slot allocation practice has been identified and explored by various studies, which implicitly or explicitly address the allocation of slots at a network of interconnected airports simultaneously taking into account slot interdependencies at departure and arrival airports. The simultaneous allocation of slots at different interconnected airports gives rise to a slot scheduling problem at a network of airports, where in addition to constraints applying to the single airport scheduling problem, any allotment of a pair of slots to a flight should be compatible with the associated flight time.

Castelli et al. (2012) proposed a mathematical model for the slot scheduling problem at network level, which aims to minimise the airlines' shift costs (an alternative notion to schedule delay) due to the deviation of allocated slots from the commercially and operationally ideal pair of departure/arrival slots (called ideal slots) for every flight. Apart from assigning departure and arrival slots to each flight, the proposed model aims at determining the corresponding flight route. The proposed model is based on the mathematical formulation proposed in Bertsimas et al. (2011) for the ATFM problem and aims to allocate a departure and an arrival slot for each flight, while simultaneously determining the corresponding route between departure and arrival airports. Each route involves a sequence of neighbouring airspace sectors connecting the origin and destination airports. By incorporating the route selection problem into the slot scheduling problem, an estimate for the flight time is made (which is set equal to the travel time of the selected route). This enables the assessment of compatibility between departure and arrival slots allocated for each flight. Any deviation from ideal slots implies cost ("shift cost") for the airlines associated to the operation of the corresponding flight. Moreover, when the route that a flight must follow from an origin to a destination airport is longer than the desired one, additional shift costs are involved. The following notation is pertinent: $A$ is the set of airlines, $F$ is the set of flights, $F_{a} \subseteq F$ is the set of flights of airline $\alpha$, and $T$ is the set of time periods. $S$ denotes the set of (en route) sectors, $S^{f} \subseteq S \cup K$ denotes the set of sectors that can be traversed by flight $f$, including the origin and destination airports, $L_{i}^{f}$ the set of sector $i$ 's subsequent sectors $\left(i \in S^{f}\right)$ and $P_{i}^{f}$ the set of sector $i$ 's preceding sectors $\left(i \in S^{f}\right)$ for flight $f$. Moreover, let $K$ denote the set of airports, $K_{k, t}$ the capacity of airport $k$ at time $t$ and $G_{j, t}^{a}$ the number of slots on which airline $a$ has Grandfather Rights (GFR) at airport $j$ at time $t$. Each flight $(f)$ is associated to a pair of origin and destination airports ( $\operatorname{orig}_{f}$, dest $f$ ) and the corresponding ideal departure and arrival slots $\left(d_{f}, a_{f}\right)$ specified by the relevant airline. Moreover, it is assumed that each flight is associated to a time interval of acceptable departure times for the origin airport and a relevant time interval of acceptable arrival times at the destination airport denoted by $T_{\text {orig }_{f}}^{f}=\left[\underline{T}_{\text {orig }_{f}}^{f}, \bar{T}_{\text {orig }_{f}}^{f}\right]$ and $T_{\text {dest }_{f}}^{f}=\left[T_{- \text {dest }_{f}}^{f}, \bar{T}_{\text {dest }_{f}}^{f}\right]$, respectively. In this work, a flight route is defined by a sequence of adjacent airspace sectors. The number of time units that flight $f$ needs to traverse sector $j$ before entering sector $j^{\prime}$ is denoted by $l_{f j j^{\prime}}$ and constitutes a major input for the associated routing problem. In any case, the total duration of a flight $(f)$ should not exceed a threshold value denoted by end $d_{f}$. Finally, deviating $\hat{t}$ time units from the ideal arrival slot of flight $f$ involves a cost $\left(c^{f}(\hat{t})\right)$, while the cost associated with an additional flight duration of $\hat{d}$ time units as compared to the ideal one for flight $f$ is denoted by $\left(c_{\text {dur }}^{f}(\hat{d})\right)$.

A key feature in the proposed mathematical formulation is related to the decision variables used for keeping track of the presence of each flight within airspace sectors over time. A set of variables $\left(w_{j, t}^{f}\right)$ are defined by (5):

$w_{j, t}^{f}= \begin{cases}1, & \text { if flight } f \text { is in airport or sector } j \text { by time } t \\ 0, & \text { otherwise }\end{cases}$

If variable $\left(w_{j, t}^{f}\right)$ takes value 1 , then the following cases may apply: (i) if $j$ is an airspace sector, then flight $(f)$ has already entered it (however, it does not mean that the flight is still in this sector at time $(t))$, (ii) if $j$ is the destination airport, then the flight has already arrived at that airport by time $(t)$, and (iii) if $j$ is the origin airport, then the flight has already departed from that airport. Thus, a flight $(f)$ enters an airspace sector $(j)$ at time $(t)$ when the $\left(w_{j, t}^{f}-w_{j, t-1}^{f}\right)$ is equal to one. The proposed mathematical model is given by (6)-(17) below (Castelli et al. 2012). 


$$
\min \sum_{f \in F} C_{f}
$$

Subject to : $\sum_{j \in\left\{\operatorname{orig}_{f}, \text { dest }_{f}\right\}} w_{j, \bar{T}_{j}^{f}}^{f}=2 \quad \forall f \in F$

$w_{\text {orig }_{f}, \underline{T}_{\text {orig }}^{f}-1}^{f}=0 \quad \forall f \in F$

$\sum_{f \in F: \text { dest }_{f}=k \vee_{\text {orig }}=k} w_{k, t}^{f}-w_{k, t-1}^{f} \leq K_{k, t} \quad \forall k \in K, \quad t \in T$

$$
\begin{aligned}
& \sum_{f \in F \backslash F_{a}: \text { dest }_{f}=k \vee_{\text {orig }}^{f}=k} w_{k, t}^{f}-w_{k, t-1}^{f} \leq K_{k, t}-G_{k, t}^{a} \quad \forall k \in K, \\
& t \in T, \quad a \in A
\end{aligned}
$$

$$
w_{j, t}^{f} \leq \sum_{i \in P_{j}^{f}} w_{i, t-l_{f i j}}^{f} \quad \forall f \in F, j \in S^{f} \backslash\left\{\operatorname{orig}_{f}\right\}, \quad t \in T
$$

$$
w_{j, t}^{f} \leq \sum_{i \in L_{j}^{f}} w_{i, t}^{f} \quad \forall f \in F, j \in S^{f} \backslash\left\{\operatorname{dest}_{f}\right\}, \quad t=\max \{T\}
$$

$$
\sum_{i \in L_{j}^{f}} w_{i, t}^{f} \leq 1 \quad \forall f \in F, j \in S^{f} \backslash\left\{\operatorname{dest}_{f}\right\}, \quad t=\max \{T\}
$$

$w_{\text {orig }_{f}, t}^{f}-w_{\text {dest }_{f}, t+e n d_{f}}^{f} \leq 0 \quad \forall f \in F, \quad t \in T$

$w_{j, t-1}^{f}-w_{j, t}^{f} \leq 0 \quad \forall f \in F, j \in S^{f}, \quad t \in T$

$w_{j, t}^{f} \in\{0,1\} \quad \forall f \in F, j \in S^{f}, \quad t \in T$

where

$$
\begin{aligned}
C_{f} & =\sum_{t \in T_{\text {dest }_{f}}^{f}} c^{f}\left(t-a_{f}\right)\left(w_{\text {dest }_{f}, t}^{f}-w_{\text {dest }_{f}, t-1}^{f}\right) \\
& +c_{\text {dur }^{f}}^{f}\left(\sum_{t \in T_{\text {dest }_{f}}^{f} t} t\left(w_{\text {dest }_{f}, t}^{f}-w_{\text {dest }_{f}, t-1}^{f}\right)\right. \\
& \left.-\sum_{t \in T_{\text {org }_{f}}^{f}} t\left(w_{\text {orig }_{f}, t}^{f}-w_{\text {orig }_{f}, t-1}^{f}\right)-\left(a_{f}-d_{f}\right)\right)
\end{aligned}
$$

The objective function (6) consists of the sum of two parts: (i) the total cost of arrival schedule delay at the destination airports and (ii) the cost of flight time deviation from the ideal flight time. Note that $\sum_{t \in T_{\text {dest }_{f}}^{f} t} t\left(w_{\text {dest }_{f}, t}^{f}-w_{\text {dest }_{f}, t-1}^{f}\right)$ in objective function (18) expresses the arrival time of flight $(f)$ at destination airport, while $\sum_{t \in T_{\mathrm{orig}_{f}}^{f}} t\left(w_{\mathrm{orig}_{f}, t}^{f}-w_{\mathrm{orig}_{f}, t-1}^{f}\right)$ expresses the corresponding departure time from the origin airport. Thus, the second part of the objective function subtracts from the estimated flight duration (arrival minus departure time) the corresponding ideal flight duration (ideal arrival minus ideal departure time). Constraint (7) implies that each flight must leave the origin airport and arrive at destination airport. Constraint (8) states that any flight $(f)$ cannot depart from the origin airport before the maximum acceptable time $\underline{T}_{\text {orig }_{f}}^{f}$. Constraint (9) requires that the number of flights that arrive at or depart from an airport $(k)$ at time interval $(t)$ should not exceed the capacity of the airport $\left(K_{k, t}\right)$ for the specific time interval. Constraint (10) imposes the relevant Grandfather Rights (GFR) constraint. Constraints (11)-(15) define the route selection problem for each flight. Constraint (11) states that any flight entering a sector $(j)$ should traverse the sector and enter a neighbouring one $(i)$ after at least $l_{f j i}$ time units. Constraint (12) implies that any flight entering a sector $(j)$ should have crossed a preceding sector $(i)$ within at least $l_{f i j}$ time units. Constraint (13) states that any transition between sectors or between sectors and airports must have been completed by the latest arrival time of the flight. Constraint (14) implies that a route for each flight $(f)$ may traverse a sector $(j)$ at most once. Constraint (15) states that the total duration of a flight should not exceed a maximum value $\left(e n d_{f}\right)$. Finally, constraint (16) implies that if a flight has arrived at a sector $(j)$ by time $t$ $\left(w_{j, t}^{f}=1\right)$, then $w_{j, t^{\prime}}^{f}=1$ for any $t^{\prime}>t$.

The model is further enhanced with a set of valid inequalities proposed in Bertsimas et al. (2011) in order to strengthen its linear relaxation. The proposed slot scheduling model belongs to the category of resource-constrained scheduling problems, and thus, it is rather unlikely to find a solution algorithm with polynomial computational complexity. Using an IP solver, it was proven feasible to solve at a reasonable amount of time (30 min of CPU time) only randomly generated instances with 2200 flights at a network of 60 airports over a time horizon of up to $5 \mathrm{~h}$. Given that the time horizon of the real-life slot scheduling problem for a network of airports spans over a time horizon of several months, the proposed model would lead to huge integer programming problems that cannot be treated by an IP solver. In a subsequent research work, the authors proposed an ant colony optimisation algorithm able to handle real-life instances of the problem (Castelli et al. 2011). A line of criticism in the model described above is that it can be applied for scheduling a set of flights only for a given day. It does not take into account explicitly the allocation of slots for a given flight over the entire scheduling season (e.g., around six months). In order to apply the proposed model in line with the current slot allocation framework, it is necessary to formulate and solve the model for the entire time horizon of the real- 
life problem. However, the proposed model does not account for series of slots, and therefore, slots allocated to the same movement over the time horizon may differ from day to day. Moreover, this model includes capacity constraints for the total number of movements per time unit without accounting for capacity constraints associated to arrivals or departures only. Finally, the model does not address the rolling capacity constraints applied at schedule coordinated airports.

Corolli et al. (2014) enriched the slot scheduling problem at network level by considering explicitly the inherent uncertainty of airport capacity. They argued that incorporating the uncertainty of actual airport capacity in the slot allocation model throughout the planning horizon may lead to substantial benefits on the basis of operational delays. In particular, Corolli et al. (2014) extended the single-airport model proposed by Zografos et al. (2012) for a network of interconnected airports, treating airport capacity as time-dependent and stochastic. A two-stage stochastic programming formulation with recourse was developed with the aim to minimise the sum of schedule delays and expected operational delays, taking into account airport capacity, flight connectivity and turnaround time constraints. In this model, the flight time between any pair of interconnected airports is assumed fixed and known in advance. By allocating a slot at the origin/destination airport, the corresponding slot at the associated destination/origin airport is fixed accordingly.

At the first stage of the proposed stochastic model, flights are scheduled by allocating slots to origin and destination airports. Given an assignment of slots to the associated requested movements, the second stage of the model aims to estimate the corresponding expected operational delay over all possible airport capacity realisations. For each possible realisation of airport capacity (modelled as random variable), Corolli et al. (2014) approximate operational delays by the total number of delayed movements (i.e., movements whose actual landing or take-off time deviates from scheduled). In particular, two alternative ways for approximating operational delays are proposed. In the first approach, the delayed movements per coordination time interval for a given airport are calculated as the difference between the number of movements requesting a slot in that interval and the corresponding airport capacity. However, delayed movements in one time interval are summed up to the allocated movements for the subsequent time interval. In order to incorporate the downstream effects of delays, the second (more accurate) approach updates the demand of the next time period with the number of flights not served due to capacity constraints in the current time period. The solution of the proposed stochastic model was achieved through the sample average approximation method. The model was applied at different interconnected airport networks including 5-10 airports. Slot requests for four different days were considered resulting to problem instances ranging from 6520 up to 9267 flights. The solution time ranged from 1.7 to almost $40 \mathrm{~h}$. Although the resulting computational times seem viable within the relevant strategic decision-making framework, solving the slot scheduling problems for a larger airport network and realworld time horizon (i.e., scheduling season) requires further investigation.

Complementing the previous work on slot scheduling models at network level, the work of Pyrgiotis and Odoni (2015)) introduces a new criterion on the slot scheduling problem that expresses the maximum displacement associated to a schedule (i.e., schedule delay). Pyrgiotis and Odoni (2015) demonstrated a demand smoothing (or flight rescheduling) model integrated with a network queuing model to estimate both local and system-wide effects of introducing scheduling limits (e.g., capacity caps) at busy airports. The demand smoothing model, formulated as an integer program, starts from a given (initial) schedule based on airlines' preferences and produces a feasible modified schedule complying with the scheduling limits applied at an airport without cancelling any flights, while simultaneously respecting all aircraft and passenger connections (i.e., all aircraft and passengers should be able to fly their original routes/itineraries). The proposed model generates a new schedule for a single day of network operations that lexicographically minimises the maximum and total displacement from the initial schedule. Unlike the model proposed by Castelli et al. (2012), the proposed model does not take into account explicitly the flight time feasibility. Instead, the model assumes that the initial flight schedule announced by airlines is feasible with respect to flight time. Based on this assumption and forcing the displacement for a departure to be equal to the displacement for the corresponding arrival, the flight time feasibility is only implicitly imposed. Although this assumption alleviates the flight time feasibility constraint from the network-based slot allocation problem, it unnecessarily excludes solutions (schedules) that might be feasible in terms of flight time, albeit with uneven departure and arrival displacement. Another limitation of the model is that it addresses the airport scheduling problem only for a single day, and hence, it cannot be directly applied for allocating slots throughout the scheduling season. The model was applied for assessing the impact of the proposed flight rescheduling on queuing delays for one of the busiest days in 2007 of Newark Liberty International airport (1910 movements). Results of the relevant computational tests demonstrated substantial reduction in queuing delays.

All models discussed above aim at supporting the coherent initial allocation of slots to airlines accommodating their flights within a network of interconnected airports. Therefore, their applicability in the existing slot allocation framework is limited to the initial allocation of slots to airlines without accounting for the possibility of slot exchanges between airlines at a post-allocation stage. Along these lines, 
Pellegrini et al. (2012) introduced a hybrid model based on the model previously proposed by Castelli et al. (2012). The model proposed by Pellegrini et al. (2012) aims to: (i) support the exchange of slots initially allocated to airlines and (ii) allocate slots to new requests issued by airlines at the secondary phase of the slot allocation process. The work presented in Pellegrini et al. (2012) is based on the assumption that the existing bilateral slot exchange framework is replaced by a market framework operating as a combinatorial exchange mechanism (Sandholm et al. 2002). The implementation of the proposed market framework is facilitated by a deterministic slot scheduling model. The proposed model is formulated as a linear integer program aiming to allocate slots at both origin and destination airports of each flight by minimising shift costs (another term for schedule delay used in literature) on the basis of initial allocation of slots to flight requests.

The model is defined on a network of interconnected airports $(K)$ operating a set of flights $(F)$ of a set of airlines $(A)$ through airspace partitioned to a set of sectors $(S)$. The notion of slot is extended for the airspace sectors, expressing a time interval in which a sector can be entered by a flight. Instead of allocating departure, arrival and airspace sector slots to flight requests, as in the model proposed for the initial slot allocation phase, the model for the secondary phase assigns a bundle of slots defining a flight route to each flight request. In this context, any feasible solution to the model involves a bundle of slots assigned to the departure and arrival airports along with the airspace sectors defining a feasible route between the given pair of airports. The objective of the proposed model is to assign a feasible route to each of the flights so that the total shift cost for the airlines is minimised subject to: (i) grandfather rights constraints, (ii) capacity constraints for airports and airspace sectors, (iii) neutrality of the revenue scheme (i.e., total amount paid or received by airlines equals zero) and (iv) airline-specific maximum acceptable value of total cost for airlines (i.e., total shift cost plus the payment made by the airline on the basis of the trading mechanism). The model was tested on a small-size problem instance of 20 airlines with approximately 3000 slots at a network of 40 airports and 200 airspace sectors for a 5-h time horizon. The test problems were solved to optimality by an IP solver. The authors argue that the application of the proposed model at the secondary stage of slot allocation may lead to the reduction in airlines cost as compared to the current practice.

\section{Emerging modelling needs and future research directions}

A detailed review of the major thrust and evolution of relevant research in strategic slot scheduling has been presented in the previous section. Increasing recent research has shown that the efficiency of the allocation outcome can be substantially improved with the use of schedule optimisation techniques. Strategic slot scheduling models aim to optimally allocate slots at a single airport, with some interesting extensions to the network problem appearing quite recently in the literature. Existing models apply the IATA-based scheduling rules in varying degrees and with several adaptations or assumptions, including single or multiple objectives, and various types of operational or regulatory constraints. The analysis of real-world attributes and decision-making requirements of the scheduling problem in conjunction with the capabilities and features offered by existing scheduling models may lead to a classification scheme (Fig. 6) according to the following criteria:

- Geographical coverage: problems are primarily classified according to their geographical coverage in terms of allocating slots at a single airport or (part of) the airport network. Depending on the level of aggregation in estimating the associated flight times, airport network models are further categorised into aggregate models, where ad hoc values are used for flight times, and disaggregate models with flight times being specified on the basis of the selection of a given flight route. Network models basically introduce extensions to single-airport models. However, obtaining exact solution in reasonable time for real-world problem instances proves to be quite challenging. Furthermore, there should be a thorough investigation of impacts of modelling complexity on the viability and accuracy of the solution approach.

- Number of optimisation criteria: it deals with the selection of the suitable number of optimisation criteria and classifies the problems into those addressing a single objective versus those pursuing the optimisation of multiple objectives.

- Objective function: another criterion relates to the type of objectives addressed or the different expressions / forms of the objective function. Existing models have primarily focused on the minimisation of a delay-based (e.g., schedule delay, queuing delay) cost function. Other objectives (e.g., costs, resource utilisation, fairness and equity, market access and competition, emissions) or variations of existing objectives (e.g., slot rejections due to unacceptable schedule delay based on maximum acceptable thresholds) may be also explored. Furthermore, alternative expressions of schedule delay (e.g., schedule delay variance, stepwise linear cost functions) introduce an interesting direction for future research.

- Capacity nature: an important factor is the modelling of capacity as a deterministic or stochastic variable. Given the strategic horizon of the scheduling problem at hand, deterministic capacity can be reasonably accepted for medium-term planning purposes. On the other hand, sto- 


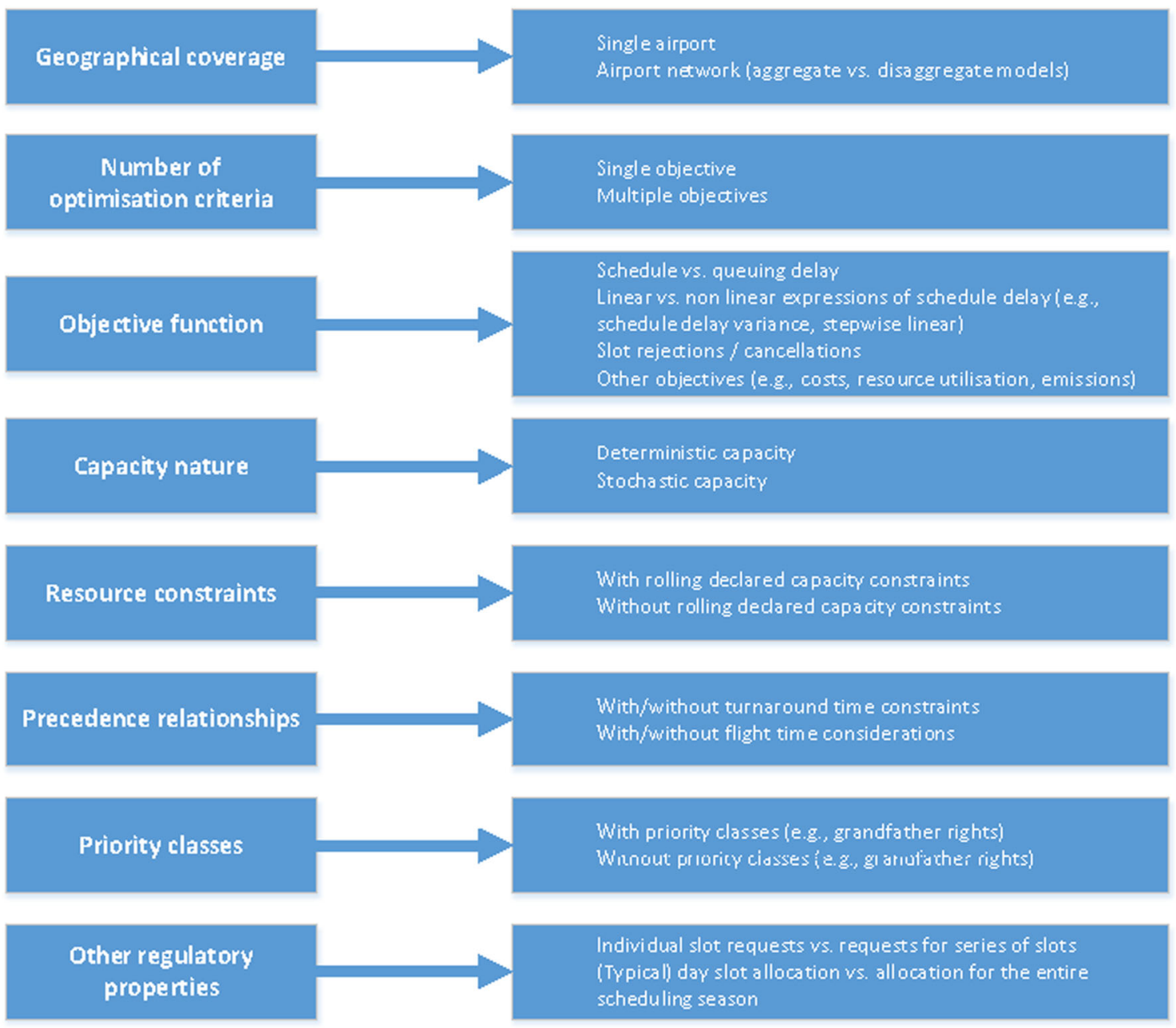

Fig. 6 Classification scheme of scheduling problems for strategic airport slot allocation

chastic capacity modelling (e.g., due to weather-induced variability) would be able to better match scheduling decisions and promote consistency among various ATM planning stages, ranging from strategic airport slot allocation to tactical flow management.

- Resource constraints: declared capacity represents the main resource constraint, while the setting of optimal declared capacity level merits further research. These constraints are often expressed in the form of rolling capacity constraints with the aim to control traffic concentration in different time intervals. The application of rolling capacity constraints is dictated by the existing regulatory regime. Nevertheless, they substantially affect the formulation, and most importantly, the complexity and accuracy of the scheduling problem.

- Precedence relationships: these are basically sequencing constraints ensuring flight connectivity. This is often achieved by means of turnaround time constraints of the paired flight legs (i.e., arrival, departure) and flight connectivity constraints among interconnected airports.
- Priority classes: both the model formulation and solution approach vary substantially with the incorporation (or not) of different priority classes (with explicit priority to historical slot holdings or "grandfather rights"). An interesting research challenge will be to explicitly consider different priority classes in the model formulation, while at the same time, pursuing solution approaches for all priority classes simultaneously.

- Other regulatory properties: models can also differ with the degree of simplification or conformance to certain regulatory properties. Such typical properties are the allocation of individual slots versus series of slots and the allocation of slots for a single day versus the entire scheduling season. Future scheduling models should be able to allocate series of slots throughout the entire scheduling season.

Figure 6 provides a schematic illustration of the classification of existing scheduling models for strategic airport slot allocation. Based on this classification, we aim to identify open 
issues and gaps that should be further investigated in future research with the main emphasis placed on the two most prominent research themes: (i) single-airport slot scheduling and (ii) network-based slot scheduling.

\subsection{Single-airport slot scheduling}

It is interesting to see that although many researchers have already started exploring the network-based slot scheduling problem, there is still ample room for further research and improvements at the single-airport level. The underlying motivation is twofold: (i) deploy a more realistic, comprehensive and viable modelling and solution approach and (ii) further investigate and improve the way that airlines' preferences are incorporated in the slot scheduling process.

The next generation of slot scheduling models should employ objectives that are currently addressed in existing literature (e.g., queuing delay, schedule delay), enriched with fairness and equity considerations (e.g., airports or cities served to safeguard connectivity to small communities, slots reserved for established airlines and new entrants), resource utilisation objectives (e.g., number of unused slots due to large deviation from requested slots), as well as environmental priorities (e.g., total or average noise exposure or emissions). Alternative formulations or expressions of objectives that are already used in existing literature merit also some further exploration. An illustrative example is schedule delay that has been treated in literature as a proxy of the utility or dis-utility that an airline encounters due to schedule displacement. For simplicity, schedule delays have been modelled in existing literature as linear cost functions expressing the difference between requested and allocated slot times. Other forms of objective functions may be also considered. For instance, taking into account the "time buffer" (or schedule padding) that airlines artificially incorporate into their flight schedules, it would be interesting to assign different weights to positive deviations (slot allocated later than requested) as compared to negative deviations (slot allocated earlier than the requested slot time). Nonlinear forms, like quadratic penalties for deviation from requested times or step-wise linear approximations, would be also an interesting area for research. In a similar context, a measure of variance of the deviation between allocated and requested slots could be potentially explored as an aggregate metric of the overall schedule acceptability. Speaking about schedule acceptability, alternative cost functions may account for thresholds on unacceptable displacement of requested slots (above which slots are practically not operated by airlines). Alternatively, the minimisation of the maximum displacement (schedule delay) can be pursued as a measure of the worst-case service level provided to airlines. At the outset, future modelling efforts should aim to obtain better insight into airlines' utility (or dis-utility) of alternative slot alloca- tion outcomes, including measures and levels of tolerance against deviations from requested slot times.

The incorporation of the above modelling issues in the basic single airport slot allocation problem will give rise to a new category of resource-constrained scheduling models with the following features: (i) multiple, start time-dependent objective functions that are non-regular (i.e., non-monotonically increasing with respect to finish times) (Sprecher et al. 1995) and (ii) time window constraints per slot request. Given that at least one of the objective functions of the problem (schedule delay) is non-regular, searching for the optimal solution in the subset of active schedules is no longer valid, and thus, a search in a wider solution space is essential (Sprecher et al. 1995). Sequential and parallel list-based constructive heuristic algorithms developed (Kolisch and Hartmann 1999, 2006) for the general resource-constrained problem can also be used for the emerging scheduling models. A key challenge in these types of heuristics relates to enhancements required in order to check efficiently the additional resource and scheduling constraints for each constructed schedule. Moreover, various metaheuristic methods already used for the general resourceconstrained scheduling problem could also be applied, such as the Tabu search (Valls et al. 2003), Simulated Annealing (Bouleimen and Lecocq 2003), population-based heuristics (Hartmann 2002) and Ant Colony Optimization (Merkle et al. 2002).

Math-based heuristics such as linear and Lagrangian relaxations or primal dual-based heuristics may also constitute a valid direction in developing solution approaches. It is notable to point out that relaxing the capacity constraint from the resource-constrained scheduling problem (with regular or non-regular start time-dependent objective function) yields a scheduling problem that can be efficiently solved in polynomial time (Möhring et al. 2003). This result implies that Lagrangian relaxation heuristics provide a promising option in solving the emerging new generation slot allocation models. Moreover, the successful implementation of constraint propagation techniques in addressing the flight gate scheduling problem (Dorndorf et al. 2007) implies that it is worth studying the effect of these techniques on the solution space of the emerging slot scheduling models.

\subsection{Network-based slot scheduling}

Building on the aforementioned improvements or extensions of single airport models, scheduling models for the airport network should aim to effectively capture strong slot complementarity issues at the airport network level. The schedules of the slot requests at the airports of a network are interdependent since assigning a departure interval time at the origin (or destination) airport of a flight constrains the arrival time interval that is assigned at the arrival (or departing) airport. 
Thus, determining feasible slot schedules at each airport of the network is not sufficient to create a feasible master slot schedule for the airport network as a whole. The flight time between airports plays a key role in checking the feasibility of the master schedule. Assigning ad hoc values for the flight time between each pair of connected airports renders the slot allocation problem at network level a resource-constrained scheduling problem, where jobs are the slot requests at the airports of the network, each flight is modelled as a precedence constraint on the relevant arrival and departure slots, and the airport capacity constitutes the available resource units.

Categories of models and algorithms mentioned for the single-airport slot scheduling problems also apply in this problem. However, a more accurate estimation of flight time involves the simultaneous determination of the route in the ATM system (e.g., airspace sectors) for each flight. The emerging route selection problem aims to assign a route to each flight taking into account: (i) the airspace sector capacities, (ii) en route congestion and (iii) alternative flight routes. The emerging route selection problem was identified as a network flow problem and explicitly incorporated in the model proposed by Castelli et al. (2012). Moreover, the existing work on slot scheduling at network level raises the following concerns regarding its real-world applicability: (i) the detailed consideration of the route selection problem has given rise to huge integer programming models solvable within reasonable time (even by heuristics) only for a time horizon of a few hours and (ii) flight and passenger connectivity constraints are not taken into account. In addition, future modelling improvements should explicitly consider the allocation of series of (rather than individual) slots and the allocation of slots according to different priority classes (e.g., grandfathered, new entrants), which have not been explicitly addressed by existing airport network models.

Most importantly, the focus of future research should be placed on the generation of alternative robust slot scheduling models and solution approaches being able to deal with the size and complexity of slot allocation at network level. As a matter of fact, the computational performance results of exact methods used to deal with small to medium-sized problem instances of slot scheduling models are not encouraging in using exact algorithms to solve to optimality real-life problem instances. Trade-offs between modelling complexity and solution accuracy certainly need to be investigated. The complexity and size of the resulting scheduling problem triggers the need for the development of decomposition schemes. Dealing with the slot scheduling problem through a hierarchy of stages (e.g., first allocate slots at hub airports, then deal with slots at remaining "satellite" airports) represents an indicative example. In any case, heuristic algorithms are needed to deal with real-life instances of the slot scheduling problem at network level. Heuristic algorithms that have been successfully applied in resource-constrained scheduling problems include: (i) Tabu Search algorithms (Thevenin et al. 2015), (ii) evolutionary algorithms (Datta et al. 2008) and (iii) Ant Colony optimisation algorithms (Castelli et al. 2011).

\section{Concluding remarks}

The strategic scheduling of airport slots signifies a challenging stream of research that has drawn the increasing attention of the research community during the last decade or so. Its main advantages stem largely from its potential to deliver quick capacity utilisation improvements, while simultaneously dealing with the associated complexity and size of the resulting mathematical problems. A critical issue in slot scheduling pertains to the determination of declared airport capacity, which essentially specifies the number of slots available for allocation. Therefore, slot scheduling and the setting of optimum declared capacity levels are closely interdependent and both lie at the heart of optimising the allocation and use of scarce airport resources. The analysis of emerging decision-making requirements vis-à-vis the capabilities and features provided by existing slot scheduling models led to the identification of open issues and gaps that should be further investigated in future research. The paper concludes with the development of concrete directions for the two most prominent streams of research related to declared capacity and slot allocation modelling. The latter topic is further analysed with view to modelling and solving both single-airport and network-based slot allocation problems.

Future research in slot scheduling should also aim to drive towards a more "holistic model" that will be able to investigate all types of interactions including: (i) propagation effects moving from single airport to the airport network and vice versa, (ii) interlinking between the various ATM planning stages (i.e., strategic, tactical, operational), (iii) multiple impacts of post-allocation activity (e.g., operational delays, resource utilisation, noise, emissions, costs) and (iv) strong interdependencies in allocating different resources (e.g., runway slots, en route slots, apron capacity, terminal capacity, ground handling equipment) even at the same allocation phase.

An interesting extension or complement to the slot scheduling approaches is offered by market-based capacity allocation mechanisms. A number of options for reforms to the EU Regulation on Slot Allocation have been considered, including the introduction of market-based mechanisms for primary (e.g., auctions) and secondary allocation of slots (e.g., secondary trading, partial auctioning of the slot pool). The common denominator of all market-based options is that they employ different methods in order to develop a better 
understanding of real market (or approximations of) valuations of slots by airlines. The real value of performing an operation at a congested airport provides critical input to the slot scheduling problem in setting appropriate scheduling levels (through declared capacity determination) and allocating efficiently slots to those valuing them most. A challenging direction for future research lies on the interface between scheduling and economic models. Ideally, future scheduling models will be able to incorporate and explicitly model, rather than implicitly consider through alternative cost expressions of schedule delay, the real economic valuations that airlines assign on scarce airport slots.

Acknowledgements The work reported in this paper has been supported by the UK's Engineering and Physical Sciences Research Council (EPSRC) through the Programme Grant EP/MO20258/1 "Mathematical models and algorithms for allocating scarce airport resources (OR-MASTER)".

Open Access This article is distributed under the terms of the Creative Commons Attribution 4.0 International License (http://creativecomm ons.org/licenses/by/4.0/), which permits unrestricted use, distribution, and reproduction in any medium, provided you give appropriate credit to the original author(s) and the source, provide a link to the Creative Commons license, and indicate if changes were made.

\section{References}

Airport Council International (ACI) Europe. (2004). Study on the use of airport capacity (pp. 1-15). Belgium: Brussels.

Airport Council International (ACI) Europe. (2009). ACI Europe position on the proposed revision of the Council Regulation (EEC) No 95/93 on common rules for the allocation of slots at Community airports. Presentation at the TRAN Meeting at the European Parliament, March 25, Strasbourg, France.

Airports Commission. (2013). Interim report. http://www.gov.uk/ government/uploads/system/uploads/attachment_data/file/27123 1/airports-commission-interim-report.pdf. Accessed December 22, 2014.

Andersen, K., Cornuéjols, G., \& Li, Y. (2005). Reduce-and-split cuts: Improving the performance of mixed-integer Gomory cuts. Management Science, 51(11), 1720-1732.

Andreatta, G., \& Lulli, G. (2009). Equitable demand management strategies for different classes of customers. International Journal of Pure and Applied Mathematics, 57(1), 1-22.

Andreatta, G., Odoni, A. R., \& Richetta, O. (1995). Models for the ground holding problem. In A. R. Odoni \& L. Bianco (Eds.), Large scale computation and information processing in air traffic control (pp. 125-168). Berlin: Springer.

Ball, M., Barnhart, C., Nemhauser, G., \& Odoni, A. (2007). Air transportation: Irregular operations and control. Handbook in Operations Research \& Management Science, 14, 1-67.

Barnhart, C., Fearing, D., Odoni, A. R., \& Vaze, V. (2012). Demand and capacity management in air transportation. EURO Journal of Transportation and Logistics, 1(1-2), 135-155.

Bennell, J. A., Mesgarpour, M., \& Potts, C. N. (2013). Airport runway scheduling. Annals of Operations Research, 204(1), 249-270.

Bertsimas, D., Lulli, G., \& Odoni, A. (2011). An integer optimization approach to large-scale air traffic flow management. Operations Research, 59(1), 211-227.
Bianco, L., Dell' Olmo, P., \& Giordani, S. (1997). Scheduling models and algorithms for TMA traffic management. In L. Bianco, P. Dell' Olmo, \& A. R. Odoni (Eds.), Modelling and simulation in air traffic management (pp. 139-167). Berlin: Springer.

Bouleimen, K., \& Lecocq, H. (2003). A new efficient simulated annealing algorithm for the resource-constrained project scheduling problem and its multiple mode version. European Journal of Operational Research, 149(2), 268-281.

Brucker, P., Drexl, A., Möhring, R., Neumann, K., \& Pesch, E. (1999). Resource-constrained project scheduling: Notation, classification, models, and methods. European Journal of Operational Research, 112(1), 3-41.

Brueckner, J. K. (2002). Internalisation of airport congestion. Journal of Air Transport Management, 8(3), 141-147.

Burke, E. K., Jackson, K. S., Kingston, J. H., \& Weare, R. F. (1997). Automated university timetabling: The state of the art. The Computer Journal, 40(9), 565-571.

Burke, E. K., Petrovic, S., \& Qu, R. (2006). Case-based heuristic selection for timetabling problems. Journal of Scheduling, 9(2), $115-132$.

Caprı, S., \& Ignaccolo, M. (2004). Genetic algorithms for solving the aircraft-sequencing problem: The introduction of departures into the dynamic model. Journal of Air Transport Management, 10, 345-351.

Castelli, L., Pellegrini, P., \& Pesenti, R. (2011). Ant colony optimization for allocating airport slots. In 2nd International conference on models and technologies for ITS (MT-ITS), Leuven, Belgium, June 22-24.

Castelli, L., Pellegrini, P., \& Pesenti, R. (2012). Airport slot allocation in Europe: Economic efficiency and fairness. International Journal of Revenue Management, 6(1/2), 28-44.

Christofides, N., Alvarez-Valdes, R., \& Tamarit, J. M. (1987). Project scheduling with resource constraints: A branch and bound approach. European Journal of Operational Research, 29(3), 262273.

Churchill, A. M., Lovell, D. J., Mukherjee, A., \& Ball, M. O. (2012). Determining the number of airport arrival slots. Transportation Science, 47(4), 526-541.

Cohen, D. A., \& Odoni, A. R. (1985). A survey of approaches to the slot allocation problem. Report R85-3, Flight Transportation Laboratory, Massachusetts Institute of Technology.

Corolli, L., Lulli, G., \& Ntaimo, L. (2014). The time slot allocation problem under uncertain capacity. Transportation Research Part C-Emerging Technologies, 46, 16-29.

Datta, D., Fonseca, C. M., \& Deb, K. (2008). A multi-objective evolutionary algorithm to exploit the similarities of resource allocation problems. Journal of Scheduling, 11, 405-419.

De Neufville, R., \& Odoni, A. R. (2003). Airport systems: Planning, design, and management (1st ed.). New York: McGraw-Hill.

Dorndorf, U., Drexl, A., Nikulin, Y., \& Pesch, E. (2007). Flight gate scheduling: State-of-the-art and recent developments. Omega, 35, 326-334.

Dorndorf, U., Jaehn, F., \& Pesch, E. (2016). Flight gate assignment and recovery strategies with stochastic arrival and departure times. $O R$ Spectrum. doi:10.1007/s00291-016-0443-1.

Eurocontrol. (2005). Report on punctuality drivers at major European airports. Report commissioned by the Performance Review Commission (PRC), Brussels, Belgium.

Eurocontrol. (2009). ATM Airport Performance (ATMAP) framework: Measuring airport airside and nearby airspace performance. Report commissioned by the Performance Review Commission (PRC), Brussels, Belgium.

Eurocontrol. (2013). Centralised Service (CS1) on Flight Plan and Airport Slot Consistency (FAS). Concept of Operations (CONOPS) Report, Edition 2.0, Brussels, Belgium. 
Eurocontrol. (2014). CODA digest: Delays to air transport in Europe (Annual 2013). Report prepared by Eurocontrol's Central Office for Delay Analysis (CODA), Brussels, Belgium.

European Commission. (1993). European Council Regulation No. 95/93 of January 1993 on Common Rules for the allocation of slots at community airports (pp. 1-6). Brussels: Official Journal of the European Union, L014.

European Commission. (2004). European Council Regulation No 793/2004 of April 2004 amending Council Regulation No 95/93 on common rules for the allocation of slots at Community airports (pp. 50-60). Brussels: Official Journal of the European Union, L138.

European Commission. (2009). European Council Regulation No 545/2009 of June 2009 amending Council Regulation No 95/93 on common rules for the allocation of slots at Community airports (pp. 24-25). Brussels: Official Journal of the European Union, L167.

European Commission. (2011). (Airport package) Proposal for a regulation of the European Parliament and of the council on common rules for the allocation of slots at community airports. $\mathrm{COM(2011)}$ 827 final, Brussels, Belgium.

European Commission. (2013). EU transport in figures. Statistical pocketbook. ISBN 978-92-79-28860-9, Belgium.

Fisher, J. B. (1989). Managing demand to reduce airport congestion and delays (vol. 1218, pp. 1-10). Washington, DC: Transportation Research Record (TRR), Transportation Research Board (TRB), National Research Council.

Garey, M. R., \& Johnson, D. S. (1979). Computers and intractability: A guide to the theory of NP-completeness. New York: W.H. Freeman.

Haghani, A., \& Chen, M.-C. (1998). Optimizing gate assignments at airport terminals. Transportation Research Part A-Policy and Practice, 32(6), 437-454.

Hartmann, S. (2002). A self-adapting genetic algorithm for project scheduling under resource constraints. Naval Research Logistics, 49, 433-448.

International Air Transport Association. (IATA). (2014a). Fact sheet: Single European Sky (SES). http://www.iata.org/pressroom/facts_ figures/fact_sheets/pages/ses.aspx. Accessed May 20, 2014.

International Air Transport Association (IATA). (2014b). Worldwide slot guidelines (6th ed.), Montreal, Canada.

Jacquillat, A., \& Odoni, A.R. (2015). An integrated scheduling and operations approach to airport congestion mitigation. Operations Research, 1390-1410, Published Online December 31, 2015. doi:10.1287/opre.2015.1428.

Koesters, D. (2007a). Study on the usage of declared capacity at major German airports. Study in cooperation between RWTH Aachen University and Eurocontrol Performance Review Unit (PRU).

Koesters, D. (2007b). Airport scheduling performance-An approach to evaluate the airport scheduling process by using scheduled delays as quality criterion. In Proceedings of the Air Transport Research Society (ATRS) annual world conference, June 21-23, Berkeley, USA.

Kolisch, R., \& Hartmann, S. (1999). Heuristic algorithms for solving the Resource-Constrained Project Scheduling Problem: classification and computational analysis. In J. Weglarz (Ed.), Handbook on recent advances in project scheduling. Dordrecht: Kluwer.

Kolisch, R., \& Hartmann, S. (2006). Experimental investigation of heuristics for resource-constrained project scheduling: An update. European Journal of Operational Research, 174(1), 23-37.

Long, D., Hasan, S., Trani, A.A., \& McDonald, A. (2009). Catalog of Models for Assessing the Next-Generation Air Transportation System. LMI Report NS802T2 for NextGenAirportal Project, Logistics Management Institute (LMI).

Lucic, P., Ohsfeldt, M., Rodgers, M., \& Klein, A. (2007). Airport runway capacity model review. Research Report by CSSI and Air
Traffic Analysis, Inc. for FAA ATO-P Performance Analysis and Strategy.

Madas, M. A., \& Zografos, K. G. (2006). Airport slot allocation: From instruments to strategies. Journal of Air Transport Management, 12(2), 53-62.

Madas, M. A., \& Zografos, K. G. (2008). Airport capacity vs. demand: Mismatch or mismanagement? Transportation Research Part APolicy and Practice, 42(1), 203-226.

Madas, M. A., \& Zografos, K. G. (2010). Airport slot allocation: A time for change? Transport Policy, 17(4), 274-285.

Malone, K. M. (1995). Dynamic queuing systems: Behavior and approximations for individual queues and networks. Doctoral Dissertation, Operations Research Center, Massachusetts Institute of Technology, Cambridge, MA.

Merkle, D., Middendorf, M., \& Schmeck, H. (2002). Ant colony optimization for resource-constrained project scheduling. IEEE Transactions on Evolutionary Computation, 6, 333-346.

Möhring, R., Schulz, A., Stork, F., \& Uetz, M. (2003). Solving project scheduling problems by minimum cut computations. Management Science, 49(3), 330-350.

Morrison, S., \& Winston, C. (2007). Another look at airport congestion pricing. American Economic Review, 97(5), 1970-1977.

Mott MacDonald. (2006). Study on the impact of the introduction of secondary trading at community airports (vol. I), Technical Report for the European Commission (DG TREN).

Narciso, M. E., \& Piera, M. A. (2015). Robust gate assignment procedures from an airport management perspective. Omega, 50, 82-95.

National Economic Research Associates (NERA). (2004). Study to assess the effects of different slot allocation schemes. Technical Report prepared for the European Commission (DG TREN), London, UK.

Odoni, A. R., \& Morisset, T. (2010). Performance comparisons between US and European airports. In Proceedings of the 12th world conference on transport research (WCTR), July 11-15, 2010, Lisbon, Portugal.

Office of Technology Assessment (OTA). (1984). Airport system development, OTA-STI-231 (pp. 109-120). Washington, DC: U.S. Congress.

Papadakos, N. (2009). Integrated airline scheduling. Computers \& Operations Research, 36, 176-195.

Pellegrini, P., Castelli, L., \& Pesenti, R. (2012). Secondary trading of airport slots as a combinatorial exchange. Transportation Research Part E-Logistics and Transportation Review, 48(5), 1009-1022.

Pita, J. P., Adler, N., \& Antunes, A. P. (2014). Socially-oriented flight scheduling and fleet assignment model with an application to Norway. Transportation Research Part B-Methodological, 61, $17-32$.

PricewaterhouseCoopers (PwC). (2000). Study of certain aspects of Council Regulation 95/93 on common rules for the allocation of slots at community airports. Technical report prepared for the European Commission.

Pritsker, A. A., Watters, L. J., \& Wolfe, P. M. (1969). Multi-project scheduling with limited resources: A zero-one programming approach. Management Science, 16, 93-108.

Pyrgiotis, N. (2011). A stochastic and dynamic model of delay propagation within an airport network for policy analysis. Ph.D. dissertation, Department of Aeronautics and Astronautics, Massachusetts Institute of Technology, Cambridge, MA.

Pyrgiotis, N., Malone, K. M., \& Odoni, A. R. (2013). Modeling delay propagation within an airport network. Transportation Research Part C-Emerging Technologies, 27, 60-75.

Pyrgiotis, N., \& Odoni, A. R. (2015). On the impact of scheduling limits: A case study at newark liberty international airport. Transportation Science. doi:10.1287/trsc.2014.0564.

Railsback, P., \& Sherry, L. (2006). A survey of rationales and methods for determining declared airport capacity. Paper presented at the 
85th Transportation Research Board annual meeting, Paper No. 06-0925.

Ranieri, A., Alsina, N., Bolic, T., Castelli, L., \& Herranz, R. (2013). Airport slot allocation: Performance of the current system and options for reform: Towards a comprehensive performance framework. In 3rd SESAR innovation days, Stockholm, Sweden.

Saleh, W. (2007). Success and failure of travel demand management: Is congestion charging the way forward? Transportation Research Part A-Policy and Practice, 41(7), 611-614.

Sandholm, T., Suri, S., Gilpin, A., \& Levine, D. (2002). Winner determination in combinatorial auction generalizations. In AAMAS 2002 proceedings of the first international joint conference on autonomous agents and multiagent systems (pp. 69-76). New York, NY: ACM.

Sprecher, A., Kolisch, R., \& Drexl, A. (1995). Semi-active, active, and non-delay schedules for the resource-constrained project scheduling problem. European Journal of Operational Research, 80, 94-102.

Stamatopoulos, M. A., Zografos, K. G., \& Odoni, A. R. (2004). A decision support system for airport strategic planning. Transportation Research Part C-Emerging Technologies, 12(2), 91-117.

Steer Davies Gleave. (2011). Impact Assessment of Revisions to Regulation 95/93. Study prepared for the European Commission (DG MOVE), London, UK.

Swaroop, P., Zou, B., Ball, M. O., \& Hansen, M. (2012). Do more us airports need slot controls? A welfare based approach to determine slot levels. Transportation Research Part B-Methodological, 46(9), 1239-1259.
Technology University of Berlin (TUB). (2001). Possibilities for the better use of airport slots in Germany and the EU. Department of Infrastructure Economics, Workgroup for Infrastructure Policy, Berlin, Germany: Technical Report prepared by the Technology University of Berlin.

Thevenin, S., Zufferey, N., \& Widmer, M. (2015). Metaheuristics for a scheduling problem with rejection and tardiness penalties. Journal of Scheduling, 18, 89-105.

Valls, V., Quintanilla, M., \& Ballestin, F. (2003). Resource-constrained project scheduling: A critical reordering heuristic. European Journal of Operational Research, 149, 282-301.

Zografos, K. G., Andreatta, G., \& Odoni, A. R. (2013). Modelling and managing airport performance. Chichester: Wiley.

Zografos, K. G., \& Madas, M. A. (2006). Development and demonstration of an integrated decision support system for airport performance analysis. Transportation Research Part C-Emerging Technologies, 14(1), 1-17.

Zografos, K. G., \& Martinez, W. (1990). Improving the performance of a port system through service demand reallocation. Transportation Research Part B-Methodological, 24(2), 79-97.

Zografos, K. G., Salouras, Y., \& Madas, M. A. (2012). Dealing with the efficient allocation of scarce resources at congested airports. Transportation Research Part C-Emerging Technologies, 21(1), 244-256. 